\title{
Weak and strong coupling regimes in plasmonic QED
}

\author{
T. Hümmer, ${ }^{1,2,3}$ F. J. García-Vidal, ${ }^{4}$ L. Martín-Moreno, ${ }^{1}$ and D. Zueco ${ }^{1,5}$ \\ ${ }^{1}$ Instituto de Ciencia de Materiales de Aragon y Departamento de Fisica de la Materia Condensada, CSIC-Universidad de Zaragoza, \\ E-50012 Zaragoza, Spain \\ ${ }^{2}$ Ludwig-Maximilians-Universität München, D-80799 Munich, Germany \\ ${ }^{3}$ Max-Planck-Institut für Quantenoptik, D-85748 Garching, Germany \\ ${ }^{4}$ Departamento de Fisica Teorica de la Materia Condensada, Universidad Autonoma de Madrid, E-28049 Madrid, Spain \\ ${ }^{5}$ Fundacion ARAID, Paseo Maria Agustin 36, E-50004 Zaragoza, Spain
}

(Received 19 December 2012; published 15 March 2013)

\begin{abstract}
We present a quantum theory for the interaction of a two-level emitter with surface plasmon polaritons confined in single-mode waveguide resonators. Based on the Green's function approach, we develop the conditions for the weak and strong coupling regimes by taking into account the sources of dissipation and decoherence: radiative and nonradiative decays, internal loss processes in the emitter, as well as propagation and leakage losses of the plasmons in the resonator. The theory is supported by numerical calculations for several quantum emitters, $\mathrm{GaAs}$ and CdSe quantum dots, and nitrogen vacancy (NV) centers together with different types of resonators constructed of hybrid, cylindrical, or wedge waveguides. We further study the role of temperature and resonator length. Assuming realistic leakage rates, we find the existence of an optimal length at which strong coupling is possible. Our calculations show that the strong coupling regime in plasmonic resonators is accessible within current technology when working at very low temperatures $(\lesssim 4 \mathrm{~K})$. In the weak coupling regime, our theory accounts for recent experimental results. By further optimization we find highly enhanced spontaneous emission with Purcell factors over 1000 at room temperature for NV centers. We finally discuss more applications for quantum nonlinear optics and plasmon-plasmon interactions.
\end{abstract}

DOI: 10.1103/PhysRevB.87.115419

PACS number(s): 42.50.Ex, 42.79.Gn, 73.20.Mf

\section{INTRODUCTION}

Cavity quantum electrodynamics (cavity QED) was invented to study and control the simplest light-matter interaction: a two-level emitter (called TLS or emitter throughout this paper) coupled to a light monomode. ${ }^{1}$ At first associated with quantum optics, the emitter was an atom or a collection of them, while the electromagnetic (EM) field was confined in a high-finesse cavity. ${ }^{2}$ Nowadays, cavity QED experiments cover quite a lot of implementations. Atoms may be replaced by other two-level systems, artificial or not, such as quantum dots or superconducting qubits. The light mode can be any single bosonic mode quantized in, e.g., superconducting cavities, ${ }^{3}$ nanomechanical resonators, ${ }^{4}$ carbon nanotubes, ${ }^{5}$ photonic cavities, ${ }^{6}$ or (collective) spin waves in molecular crystals. $^{3}$

Cavity QED relies on the comparison between the "lightmatter" coupling strength per boson and the irreversible losses from both emitter and bosonic mode. Depending on their ratio, two main regimes appear: weak and strong coupling. In the weak regime, losses dominate and the emission spectrum consists of a single peak around the dressed TLS resonant transition while the lifetime is modified because of the field confinement inside the cavity. This modification is nothing but the Purcell effect. In the strong coupling (SC) regime, the coupling dominates the losses. In this case, a double peak emerges in the emission spectrum, arising from the emitter-resonator level anticrossing. Cavity QED is interesting per se: it demonstrates the quantum nature of both light and matter, and serves, e.g., for testing quantumness in bigger and complex systems. ${ }^{7}$ But, cavity QED is also a resource, e.g., for optimizing single-photon emission ${ }^{8}$ or lasing. ${ }^{9}$ Besides, systems in the SC regime may behave as nonlinear media, ${ }^{10}$ generate photon-photon interactions, ${ }^{11}$ and are the building blocks in quantum information processing architectures. ${ }^{12}$

Although the weak coupling (WC) regime is relevant, the ultimate goal is to reach the $\mathrm{SC}$ regime. The former can be easily reached if the latter is set. Being in the SC regime is determined by the EM field lifetime, confinement, and dipole moment. Usually, when working with macroscopic mirrors and atoms having small dipole moments, the field confinement is not optimized but the cavity has extremely long lifetimes, i.e., very high finesse or quality factors. In other setups such as superconducting circuits, all parameters (quality factor, dipole moment, and field confinement) are optimized such that the socalled ultrastrong coupling regime has been demonstrated. ${ }^{13,14}$ Circuits are promising on-chip setups but have to be operated at microwave frequencies and $\mathrm{mK}$ temperatures.

A possible alternative at optical or telecom frequencies, with their plethora of applications in quantum communication, is provided by the subwavelength confined EM fields of surface plasmon polaritons (SPP) in plasmonic waveguides. ${ }^{15}$ By making resonators out of those waveguides, the energy density and therefore the coupling is highly enhanced. The payoff is that metals introduce considerable losses, further increasing with higher confinement. Therefore, it is not clear under which conditions SC could be reached with plasmonic resonators. On the other hand, advanced architectures of plasmonic waveguides present a good tradeoff between confinement and losses, ${ }^{16}$ e.g., hybrid, ${ }^{17}$ wedge, ${ }^{18}$ or channel ${ }^{19}$ waveguides. Plasmonic waveguides have already shown remarkable properties, such as focusing, ${ }^{20,21}$ lasing, ${ }^{22}$ superradiance, ${ }^{23}$ mediators for entanglement between qubits, ${ }^{24}$ and single-plasmon emission. ${ }^{8,25}$ Still, a very challenging perspective is their use for achieving quantum cavity QED 
with plasmons in the SC regime. ${ }^{26}$ Plasmonic QED is not just another layout for repeating what has been done in other cavity QED implementations but offers interesting advantages. As shown in this paper, SC can be obtained inside nanometric resonators. It can be mounted on a chip in combination with dielectric waveguides. The latter have minor losses but weakly interact with quantum emitters.

This paper aims to be self-contained. We first summarize the quantum theory for the coupling between quantum dipoles and resonators made out of one-dimensional (1D) plasmonic waveguides. Within this theoretical framework, we properly include the losses and map to a Jaynes-Cummings model and, therefore, to the physics and applications of traditional cavity QED. We present extensive finite-element simulations for a variety of resonator layouts and several quantum emitters. Our simulations allow us to set the conditions to reach the SC regime. We also motivate the study of these systems in the less demanding WC regime because of very high achievable Purcell factors into the plasmon channel of more than 1000 . We use our simulations for explaining the numbers provided in a recent experiment for quantum resonators in the WC regime. ${ }^{8}$

The paper is organized as follows. We first develop in Sec. II the light-matter interaction in plasmon resonators within the Green's function approach. In Sec. III, the different realizations for plasmonic resonators and emitters are discussed. We continue in Sec. IV with numerical results setting the parameter landscape for WC and SC regimes. Section V is devoted to emphasize different applications. Some technical details are discussed in the Appendixes.

\section{INTERACTION OF A PLASMONIC STRUCTURE AND AN EMITTER}

\section{A. Green's function approach for dissipative field quantization}

Surface plasmon polaritons ("SPPs" or just "plasmons") are surface wave quanta bound to the interface between two media characterized by permittivities $\left[\epsilon(\omega)=\epsilon^{\prime}(\omega)+i \epsilon^{\prime \prime}(\omega)\right]$ with real parts of different signs and negative sum. Usually, the interface separates a dielectric $\left[\epsilon^{\prime}(\omega)>0\right]$ and a metal, which presents $\epsilon^{\prime}(\omega) \ll 0$ at optical frequencies (see, e.g., Ref. 27). On the other hand, the imaginary part $\epsilon^{\prime \prime}(\omega)$ is responsible for dissipation in the metal (in order to minimize this dissipation, commonly used metals are silver and gold). Complex permittivities can be easily incorporated in the macroscopic Maxwell equations. However, a problem arises when trying to quantize the EM field: Maxwell equations with a complex permittivity $\epsilon^{\prime \prime}(\omega) \neq 0$ can not be obtained from a Lagrangian and, consequently, a straightforward canonical quantization is not possible. On the other hand, (linear) dissipation can be modeled by coupling the EM field to an additional bath of harmonic oscillators: the system-bath approach. ${ }^{28}$ Importantly, the system and bath can be cast to a total Lagrangian and consequently this allows the quantization of the EM field in dispersive media. ${ }^{29,30}$ For self-completeness, we outline this theory in Appendix A. To apply this quantization to complex geometries (needed for plasmonic structures) the theory can be conveniently reformulated by means of the Green's tensor of the classical problem. ${ }^{31-34}$ The usefulness of this approach can be appreciated by looking at a key result, the quantum expansion of the electric field

$$
\begin{aligned}
\vec{E}(\vec{r}, \omega)= & i \sqrt{\frac{\hbar}{\pi \epsilon_{0}}} \frac{\omega^{2}}{c^{2}} \int d^{3} r^{\prime} \sqrt{\epsilon^{\prime \prime}\left(\vec{r}^{\prime}, \omega\right)} \stackrel{\leftrightarrow}{G}\left(\vec{r}, \vec{r}^{\prime}, \omega\right) \\
& \times\left[f^{\dagger}\left(\vec{r}^{\prime}, \omega\right)-f\left(\vec{r}^{\prime}, \omega\right)\right]
\end{aligned}
$$

and an analogous expression for the magnetic field. Here, the electric field can be expanded in normal modes where the coefficients are given by the Green's function of the classical field. These normal modes of the combinedEM field and the dispersive media are represented by the bosonic creation (annihilation) operators $f^{\dagger}(\vec{r}, \omega)[f(\vec{r}, \omega)]$. They obey the commutation relation $\left[f(\vec{r}, \omega), f^{\dagger}\left(\vec{r}^{\prime}, \omega^{\prime}\right)\right]=\delta\left(\omega-\omega^{\prime}\right) \delta(\vec{r}-$ $\left.\vec{r}^{\prime}\right)$. We used $\epsilon_{0}$ to denote the vacuum permittivity and $c$ is the speed of light. Finally, $\stackrel{\leftrightarrow}{G}\left(\vec{r}, \vec{r}^{\prime}, \omega\right)$ is the dyadic Green's function of the classical field defined by ${ }^{27,33}$

$$
\left[\nabla \times \nabla \times-\frac{\omega^{2}}{c^{2}} \epsilon(\vec{r}, \omega)\right] \stackrel{\leftrightarrow}{G}\left(\vec{r}, \vec{r}^{\prime}, \omega\right)=\stackrel{\leftrightarrow}{I} \delta\left(\vec{r}-\vec{r}^{\prime}\right) .
$$

Therefore, within this formalism the quantum fields, Eq. (1), are determined by the classical Green's function, Eq. (2).

\section{B. Emitter-plasmon interaction}

We are interested in the interaction of these quantum fields with a TLS. The actual physical implementation of the emitters will be discussed in detail in Sec. III C. In the dipole approximation, the interaction can be represented by the emitter's dipole transition strength $\vec{d}$ and the electric field at the position of the emitter $\left(\vec{r}_{e}\right)$ (Ref. 35)

$$
H_{\text {int }}=-\sigma_{x} \vec{d} \cdot \vec{E}\left(\vec{r}_{e}\right),
$$

with $\vec{E}\left(\vec{r}_{e}\right)=\int_{0}^{\infty} d \omega \vec{E}\left(\vec{r}_{e}, \omega\right)$.

In plasmonic waveguides, there are instances (which require a careful positioning of the emitter) where the emitter radiates mainly into the plasmon channel. ${ }^{15,23}$ Then, we can isolate the emitter-plasmon coupling and, as explained in Ref. 36 and summarized in Appendix A, write the emitter-plasmon coupling in terms of operators that annihilate and create plasmons with frequency $\omega, a(\omega)$, and $a^{\dagger}(\omega)$, respectively. These operators have bosonic character, satisfying $\left[a(\omega), a^{\dagger}\left(\omega^{\prime}\right)\right]=$ $\delta\left(\omega-\omega^{\prime}\right)$. Then, the emitter-plasmon Hamiltonian is

$$
\begin{aligned}
H / \hbar= & \frac{\omega_{e}}{2} \sigma_{z}+\int_{0}^{\infty} d \omega \omega a^{\dagger}(\omega) a(\omega) \\
& +\int_{0}^{\infty} d \omega\left(g(\omega) \sigma^{-} a^{\dagger}(\omega)+\text { H.c. }\right) .
\end{aligned}
$$

This is the spin-boson model where the emitter, with level spacing $\omega_{e}$, is represented by standard Pauli matrices $\sigma_{x, y, z}$, $\sigma^{ \pm}=\sigma_{x} \pm i \sigma_{y}$. The coupling of the emitter to the plasmon modes is characterized by $|g(\omega)|^{2}$, also called the spectral density,

$$
|g(\omega)|^{2}=\frac{1}{\hbar \pi \epsilon_{0}} \frac{\omega^{2}}{c^{2}} \vec{d}^{T} \operatorname{Im}\left[\stackrel{\leftrightarrow}{G}_{\mathrm{spp}}\left(\omega, \vec{r}_{e}, \vec{r}_{e}\right)\right] \vec{d} .
$$

Here, $\stackrel{\leftrightarrow}{G}_{\text {SPP }}$ is the contribution of the plasmon pole to the the electromagnetic Green's tensor in the nanostructure. It contains all the information about the plasmonic structure: 
(a)

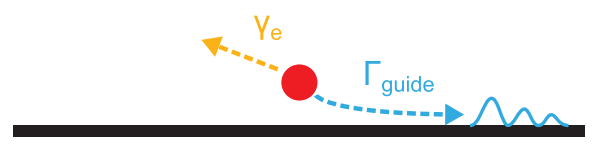

(b)

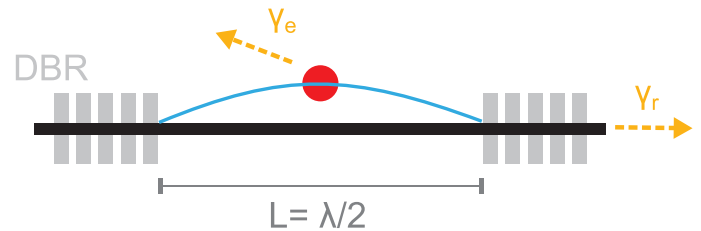

(c)

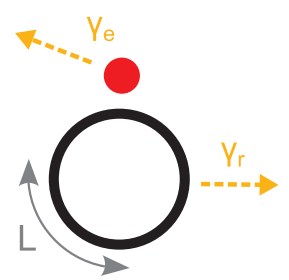

FIG. 1. (Color online) (a) Sketch of an emitter (red dot) coupled to an open plasmonic wave guide. It emits with rate $\Gamma_{\text {guide }}$ into propagating surface plasmons and with rate $\gamma_{e}$ into other modes. (b) Linear resonator defined by a waveguide enclosed by mirrors [here distributed Bragg reflectors (DBR)]. The excitations from the resonator are lost with rate $\gamma_{r}$. The length of the resonator has to be multiples of half the plasmon wavelength for resonances to occur. (c) A circular resonator configuration.

plasmon propagation length, modal shape, etc., and depends on both emitter position $\left(\vec{r}_{e}\right)$ and emitter orientation (via $\left.\vec{d}\right)$.

Equation (5) considers only the coupling between the emitter and the (lossy) plasmons. All other mechanisms for loss in the system will be introduced as Lindblad terms, which affect the nonunitary evolution of the emitter-plasmon density matrix. The origin and description of these dissipative channels will be discussed in Sec. II D.

\section{Green's function of the plasmonic structure}

A strategy for confining the EM field to a small area, and consequently enhance light-matter interaction, is by using one-dimensional metallic structures (waveguides) that support propagating plasmons. The plasmonic modes of the waveguide confine the EM field in two dimensions. Since they are mixed photon-media excitations, the confinement can exceed the one of free-space photons that is limited by diffraction. To further enhance the interaction, the plasmons can be stored in resonators. These can be manufactured out of plasmonic waveguides by placing two mirrors or building a ring, as sketched in Fig. 1.

\section{Waveguide}

Surface plasmons on an infinite ("open") waveguide can be described by $\vec{E}_{\mathrm{p}}(\vec{r})=\vec{E}_{\mathrm{p}}\left(\vec{r}_{t}\right) e^{ \pm i k(\omega) z}$, with the transverse field profile $\vec{E}_{\mathrm{p}}\left(\vec{r}_{t}\right)$, where $z$ is the coordinate parallel to the waveguide axis and $\vec{r}_{t} \equiv\{x, y\}$ is perpendicular to it. Due to propagation losses of the plasmons, their propagation constant $k(\omega)=k^{\prime}(\omega)+i k^{\prime \prime}(\omega)$ is a complex quantity. The Green's function $G_{\mathrm{SPP}}\left(\omega, \vec{r}, \vec{r}^{\prime}\right)$ can be constructed from the plasmon's electric field: ${ }^{37,38}$

$$
\stackrel{\leftrightarrow}{G}_{\mathrm{SPP}}\left(\omega, \vec{r}, \vec{r}^{\prime}\right) \approx \frac{c^{2}}{\omega v_{g}} \frac{\vec{E}_{\mathrm{p}}\left(\vec{r}_{t}\right) \otimes \vec{E}_{\mathrm{p}}^{*}\left(\vec{r}_{t}^{\prime}\right)}{\int_{A_{\infty}} d^{2} \tilde{r}_{t} \epsilon\left(\tilde{\vec{r}}_{t}\right)\left|E_{\mathrm{p}}\left(\tilde{\vec{r}}_{t}\right)\right|^{2}} k G_{1 \mathrm{D}}\left(\omega, z, z^{\prime}\right) .
$$

This is the contribution to the Green's function at the region outside the metal arising from the bound surface plasmons. In this expression, $c$ is the speed of light and $v_{g}(\omega)$ is the plasmon group velocity.

In this work, $v_{g}$ is computed numerically from the dispersion relation $v_{g}(\omega)=\partial \omega / \partial k$ using frequency-dependent values of the permittivity. ${ }^{39}$ We have found that using approximate expressions which only involve the permittivity at the working frequency may lead to incorrect results. For instance, the commonly used expression $v_{g}=\int_{A}\left(\vec{E}_{\mathrm{p}} \times\right.$ $\left.\vec{H}_{\mathrm{p}}\right)_{z} d A / \int_{A} \epsilon_{0} \epsilon(\vec{r})\left|E_{\mathrm{p}}\right|^{2} d A$, exact only for nondispersive materials, sometimes strongly overestimates the group velocity. This is especially important for the case of waveguides with highly confined modes, where the approximate expression may even predict superluminal velocities.

The expression for $G_{\mathrm{SPP}}\left(\omega, \vec{r}, \vec{r}^{\prime}\right)$ is split in a part perpendicular to the waveguide and a part along the waveguide $G_{1 \mathrm{D}}$ that matches the 1D (scalar) Green's function ${ }^{40}$

$$
G_{1 \mathrm{D}}\left(\omega, z, z^{\prime}\right)=\frac{i}{2 k} e^{i k\left|z-z^{\prime}\right|} .
$$

Evaluating the coupling strength $g(\omega)$ at the optimal position in the waveguide, using Eqs. (5) and (7) we get

$$
|g(\omega)|^{2}=\frac{1}{2 \pi} \Gamma_{0} \frac{3}{\pi \epsilon_{d}^{3 / 2}} \frac{c}{v_{g}} \frac{A_{0}}{A_{\text {eff }}} \equiv \frac{1}{2 \pi} \Gamma_{\text {guide }},
$$

where $\Gamma_{0}$ is the "free-space spontaneous emission rate" $\Gamma_{0}=$ $\sqrt{\epsilon_{d}} \omega^{3}|d|^{2} / 3 \pi \hbar \epsilon_{0} c^{3}$ for an emitter placed in a homogeneous medium with permittivity $\epsilon_{d}$ (corresponding to the permittivity of the dielectric in which the emitter is placed in). We have defined $\Gamma_{\text {guide }}$ to be the emission rate into surface plasmons of the open waveguide. The diffraction limited area $A_{0}=\left(\lambda_{0} / 2\right)^{2}$ is the minimum section in which light of wavelength $\lambda_{0}$ can be confined to in vacuum. Furthermore, we have introduced the effective mode area of the plasmon field

$$
A_{\mathrm{eff}}\left(r_{t}\right)=\frac{\int_{A_{\infty}} d^{2} \tilde{r}_{t} \epsilon\left(\tilde{\vec{r}}_{t}\right)\left|E_{\mathrm{p}}\left(\tilde{\vec{r}}_{t}\right)\right|^{2}}{\max \left\{\epsilon\left(\vec{r}_{t}\right)\left|E_{\mathrm{p}}\left(\vec{r}_{t}\right)\right|^{2}\right\}} .
$$

This magnitude is inversely proportional to the maximum energy density and therefore quantifies the achievable coupling strength.

\section{Resonator}

The 1D Green's function of a resonator can be obtained by summing over all the reflected contributions of a wave originating from a $\delta$ source. ${ }^{40,41}$ The details can be found in Appendix B. The change in the Green's function translates into a change in the spectral density, which is related but different to the spectral density in the infinite waveguide.

Here, we will look at two resonator configurations: either a linear resonator of length $L$ terminated by two mirrors with reflectivity $|R|$ or a circular resonator with circumference $L$. 


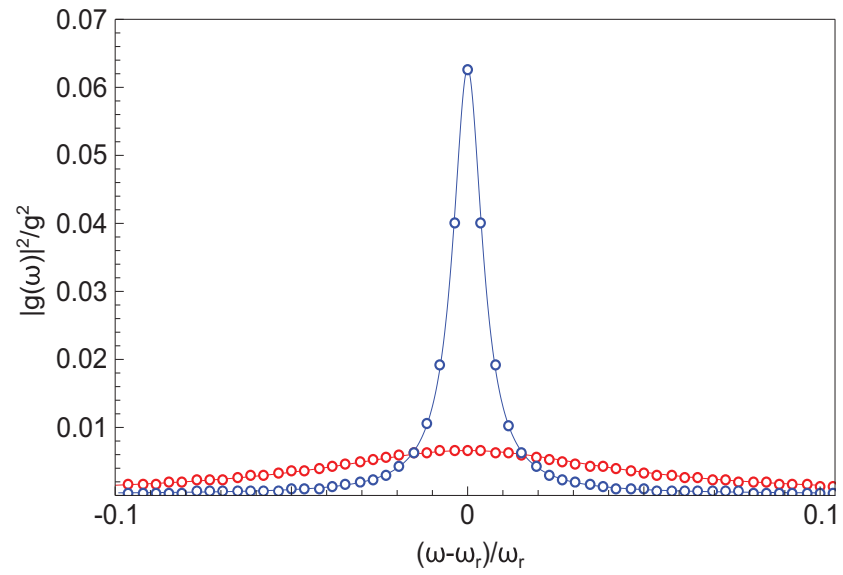

FIG. 2. (Color online) Spectral density $|g(\omega)|^{2}$ of the resonator for different losses (blue $\gamma_{r}=0.01 \omega_{r}$ and red $\gamma_{r}=0.1 \omega_{r}$ ). The solid line is the exact spectral density of a resonator following Eq. (B2), while the circles are the approximation with Eq. (12).

In both configurations, the spectral density $|g(\omega)|^{2}$ is peaked around the resonance frequencies $\omega_{r}=2 \pi v_{p} / \lambda$ as shown in Fig. 2. Here, $v_{p}$ is the phase velocity and $\lambda=\lambda_{0} v_{p} / c$ the wavelength of the SPPs. The condition for resonance is

$$
L=\frac{\lambda}{2} m=\frac{\pi v_{p}}{\omega_{r}} m,
$$

where $m$ counts the number of the field antinodes in the resonator and has to be an even integer for circular resonators and any integer for the linear configuration.

In a real system, the resonator will have losses, with different contributions that can be encapsulated in the coefficient $\gamma_{\mathrm{r}}$ :

$$
\gamma_{r} \equiv \gamma_{\text {prop }}+\gamma_{\text {leak }}=2 v_{g}\left(k^{\prime \prime}\left(\omega_{r}\right)-\frac{1}{L} \ln |R|\right),
$$

where $\gamma_{\text {prop }}$ are plasmon propagation losses and $\gamma_{\text {leak }}$ leakage through the mirrors in the linear resonator. For the circular resonator, radiative losses due to bending have to be added but will not be treated in detail here.

Taking into account losses, the spectral density can be approximated close to the resonant frequency by (see Fig. 2 and Appendix B for a derivation)

$$
|g(\omega)|^{2} \approx g^{2} \frac{2}{\pi} \frac{\gamma_{r} \omega_{r} \omega}{\left(\omega^{2}-\omega_{r}^{2}\right)^{2}+\gamma_{r}^{2} \omega^{2}},
$$

where we assumed that the resonator linewidth is small compared to the resonance position, $\gamma_{r} \ll \omega_{r}$, i.e. we have a well-defined resonance. The coupling amplitude is given by

$$
g=\sqrt{\Gamma_{\text {guide }} \frac{v_{g}}{L}} .
$$

We assumed that the emitter is positioned at a field antinode in the linear resonator to yield maximum coupling. In the circular resonator, the emitter can be placed anywhere along the waveguide.

Let us comment on the dependence $\gamma_{\text {leak }} \sim 1 / L$. Notice that $\gamma_{\text {leak }}$ is the energy loss per time through the resonator mirrors. Therefore, the leakage must be proportional to the energy density at the mirrors which is $\sim 1 / L$. In a proper resonator with highly reflective mirrors, we can expand $-\ln |R| \equiv$ $-\ln (1-|T|) \cong|T|$ for small transmission and absorption coefficients $|T| \ll 1$. Thus, the leakage is proportional to the transmittance. In contrast to the leakage, the propagation losses $\gamma_{\text {prop }}$ do not depend on the resonator length since the linewidth (and the loss rate) quantify the losses per unit of time and not per resonator roundtrip of the plasmons. The propagation losses are proportional to the imaginary part of the plasmon wave vector $k^{\prime \prime}$ or, in other words, inverse to the plasmon propagation length defined as $\ell \equiv 1 /\left(2 k^{\prime \prime}\right)$.

\section{Jaynes-Cummings model: Plasmonic QED}

We now use a mathematical result with an enormous physical relevance: the bosonic bath coupled to a TLS with a peaked spectral density $|g(\omega)|^{2}$ such as Eq. (12) can be split in a single-boson mode with frequency $\omega_{r}$ coupled to a bath characterized by a dissipation rate $\gamma_{r}$, i.e., the width of $|g(\omega)|^{2}{ }^{42-45}$ Physically, the $\omega_{r}$ mode is the single resonator mode. In the end, the plasmonic resonators discussed here can now be approximated by the Jaynes-Cummings (JC) model

$$
H_{\mathrm{JC}} / \hbar=\frac{\omega_{e}}{2} \sigma_{z}+\omega_{r} a^{\dagger} a+g\left(\sigma_{-} a^{\dagger}+\sigma_{+} a\right)
$$

with additional losses from the emitter (with rate $\gamma_{e}$ ) and from the resonator (with rate $\gamma_{r}$ ). This physics can be encoded in an optical master equation for the density matrix $\rho$, after tracing out the bath degrees of freedom. It takes the form of the celebrated Markovian Lindblad master equation ${ }^{46,47}$

$$
\begin{aligned}
\dot{\varrho}= & -\frac{i}{\hbar}\left[H_{\mathrm{JC}}, \varrho\right]+\gamma_{r}\left(a \rho a^{\dagger}-\frac{1}{2}\left\{a^{\dagger} a, \varrho\right\}\right) \\
& +\gamma_{e}\left(\sigma_{-} \varrho \sigma_{+}-\frac{1}{2}\left\{\sigma_{+} \sigma_{-}, \varrho\right\}\right)+\frac{\gamma_{d}}{4}\left(\sigma_{z} \rho \sigma_{z}-\varrho\right) .
\end{aligned}
$$

Here, we have introduced two phenomenological rates: $\gamma_{e}$ and $\gamma_{d}$, which account for a dissipative and pure dephasing channel for the emitter. ${ }^{24,48,49}$

The rate $\gamma_{e}$ accounts for all processes that provide dissipative transitions between the discrete levels of the qubit. The different contributions to $\gamma_{e}$ may be written as

$$
\gamma_{e}=\gamma_{\mathrm{rad}}+\gamma_{\mathrm{nonrad}}+\gamma_{\mathrm{int}} .
$$

Emission into free-space radiating EM modes is depicted by $\gamma_{\text {rad }}$. Furthermore, another emitter loss channel specific to plasmonic structures arises: If the emitter is placed close to a metal surface, it couples to nonpropagating, quickly decaying evanescent modes and the energy is dissipated through heating of the metal. The associated rate will be called $\gamma_{\text {nonrad }}$ and can assume very high rates when an emitter is close to a metal surface. Actually, $\gamma_{\text {nonrad }}$ (Refs. 50 and 51) is the dominant decay rate at emitter-metal distances below $\sim 10 \mathrm{~nm}$. To avoid this quenching effect, one may lift the TLS away from the metal surface and place it at an intermediate region close enough to still couple efficiently to plasmons. In all our calculations we assume that the dipole is placed at a distance of $10 \mathrm{~nm}$ from the metal surface and set $\gamma_{\text {nonrad }}=0$. This is validated from estimations of $\gamma_{\text {nonrad }}$ obtained from the fraction of energy radiated by an emitter into waveguide surface plasmons (the 
so-called " $\beta$ factor"). ${ }^{37,48}$ These estimations show that the relevant decay rate in plasmonic resonators arises from both the finite propagation of surface plasmons and the transmission at nonperfect mirrors (which is another decay route for surface plasmons in the resonator). An important point, developed in Appendix $\mathrm{C}$, is that in a plasmonic resonator the decay into nonradiative channels is penalized with respect to the decay into plasmons as the latter can be resonantly enhanced while the former can not.

Additionally, and in order to present a theory as general as possible (valid for any two-level system), we consider in Eq. (16) the possible existence of any other nonelectromagnetic dissipative channel, characterized by the phenomenological rate $\gamma_{\text {int }}$. However, the actual calculations presented in the paper are for either nitrogen vacancy (NV) centers or quantum dots, for which the dissipative rates involving transitions between discrete electronic levels are believed to be of electromagnetic origin. Therefore, in all our calculations we set $\gamma_{\text {int }}=0$.

Finally the additional phenomenological pure dephasing term $\gamma_{d}$ accounts for transitions between the vibrorotational manifold of each discrete electronic level of the qubit. Pure dephasing thus models the broadening of the spectral emission observed in solid-state emitters by, e.g., coupling to phonons. ${ }^{52-56}$

Equation (15) enables the mapping between the physics of resonators in waveguides and that of cavity QED systems. As a consequence, many of the results from Jaynes-Cummings physics in cavity QED can be exported to quantum plasmonic systems. We emphasize that, within the formalism sketched here, the master equation has been obtained from a firstprinciples theory. Therefore, parameters such as the coupling between the single-plasmon resonator mode and the emitter $g$ and the decoherence rates $\gamma_{r}$ and $\gamma_{e}$ can be computed from the emission spectra of the qubit and the Green's function of the plasmonic structure.

\section{REALIZATION OF PLASMONIC QED}

In this section, we specify the actual emitter and resonator architectures studied in this work.

\section{A. Waveguides}

The plasmon resonators treated in this work are made out of waveguides. Therefore, the final resonator properties depend critically on the specific waveguides used, especially on the achievable field confinement and plasmon propagation length. Plasmon waveguides are quasi-1D translational-invariant metal-insulator structures. They possess SPP eigenmodes that propagate along the waveguide axis, while presenting exponentially decaying evanescent fields in the transverse plane, both in the metal and in the dielectric. The waveguides we focus at may reach high-field confinements along with low propagation losses. Usually, there is a tradeoff between confinement and propagation length, but the actual values are geometrically dependent. We pick three different waveguide geometries which offer long propagation lengths along with high-field confinements, as well as good fabrication techniques: the first type are small-diameter metal
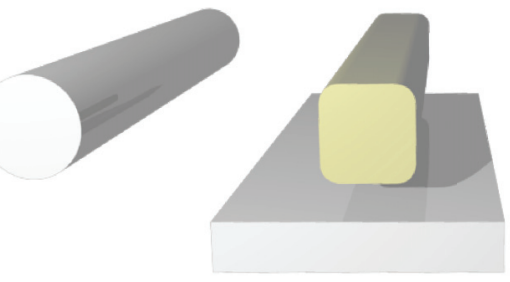

wire
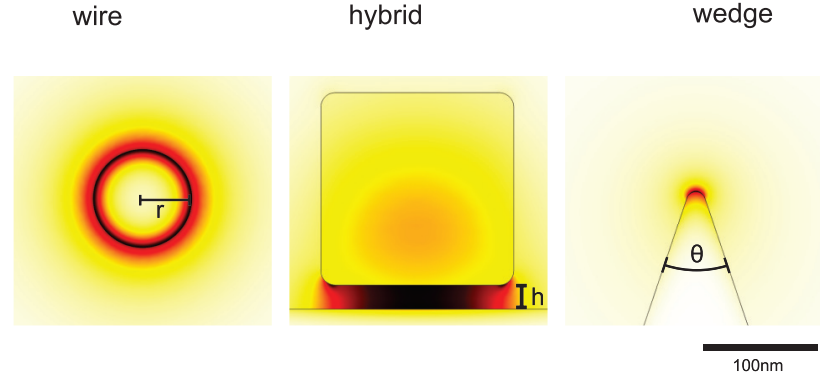

FIG. 3. (Color online) Model of the three waveguides treated in this work. Below, the relative energy density of each waveguide is sketched. The parameters used here are $r=50 \mathrm{~nm}, h=25 \mathrm{~nm}$, $\theta=40^{\circ}$, and the SPP eigenmodes are numerically computed for a frequency corresponding to a free-space wavelength.

nanowires. ${ }^{15}$ The second type are sharp metal wedges ${ }^{18,57}$ offering high-field strengths at their tips. Finally, the third class are hybrid waveguides, ${ }^{17,22}$ formed by a high refractive index dielectric nanowire (silicon $\epsilon=12.25$ ) placed close to a metal surface. There, the SPP of the plane and the mode of dielectric create a hybrid mode with strong field confinement in the gap. Transversal cuts through the three waveguides are plotted in Fig. 3 along with a sketch of their field-energy distributions. The propagation length and confinement of these waveguides depend on the concrete geometrical parameters of the waveguides. In the hybrid waveguide, the main parameter is the gap size between the dielectric and the metal surface. The nanowire properties depend on the radius and the wedge on the tip angle and the tip radius. For smaller wires, smaller gaps, or tighter angles, respectively, the field confinement increases and the propagation lengths decrease. Finally, we consider that the metal waveguides are made of silver, which offers best propagation lengths at optical and telecom frequencies and are embedded in polymethyl methacrylate (PMMA) $\left(\epsilon_{d}=2\right)$.

\section{B. Resonators}

In our calculations we consider two architectures: a circular and a linear resonator as sketched in Fig. 1.

\section{Circular resonator}

The circular resonator is formed by bending a waveguide and connecting its ends. The fundamental disadvantage is that the energy is converted from propagating modes to free radiation at bending. ${ }^{58,59}$ On the other hand, these losses decrease exponentially with increasing the radius of the ring. Moreover, it is expected that bending losses are smaller for higher confined modes. Circular waveguides can nowadays be made lithographically, but this process leads to polycrystalline structures, with its associated radiative and nonradiative losses 
at domain interfaces which impair SPP propagation. Hopefully, single-crystal circular waveguides (probably synthesized by chemical means) will be available in the near future.

\section{Linear resonator}

Linear resonators can be built by placing reflective mirrors in the waveguide. Here, scattering losses and transmission through these mirrors have to be avoided for having good resonators. Distributed Bragg reflectors were predicted to be limited to low reflectivities for plasmons on two-dimensional (2D) metal surfaces. ${ }^{60}$ However, recent resonator realizations have shown high reflectivities by combining both modes highly confined to waveguides and Bragg reflectors composed of alternating dielectric layers with small refractive index differences. ${ }^{8}$ In comparison to optical and microwave cavities, where mirror absorption, scattering, and transmission losses can be reduced to several $\mathrm{ppm},{ }^{61,62}$ plasmon mirrors are expected to exhibit losses in the order of a few percent.

\section{Quantum emitters}

Emitters should (i) be photostable, (ii) present a large dipole moment, and (iii) maintain their properties when either embedded into a solid-state substrate or placed on top of a surface. Interesting candidates are color centers in crystals or semiconductor quantum dots (QDs) grown on surfaces or chemically synthesized as nanospheres.

The emission spectrum of single atomic emitters traditionally studied in quantum optics is simply a Lorentzian with a very narrow transform-limited linewidth given by the timeenergy uncertainty relation. In contrast, solid-state emitters have higher dipole moments but are also strongly coupled to their solid-state environment. Therefore, the transform-limited line, also called zero-phonon line (ZPL), is dominated and covered by phonon sidebands, giving rise to a very broad non-transform-limited spectrum. ${ }^{52,54,56,63}$ As used in Sec. II B, this broad peak can be modeled phenomenologically by an additional source of dephasing in the master equation (15). ${ }^{52-56}$ At lower temperature, the phonon sidebands mostly vanish and the ZPL can be observed.

The first emitter we consider is the nitrogen vacancy (NV) center in diamond. ${ }^{64}$ Single NV centers can be found embedded in diamond nanocrystals of sizes down to a few $\mathrm{nm}$. At room temperature (RT), these centers are highly stable and present an emission line at $\approx 670 \mathrm{~nm}$, with a spectral width (FWHM) of $\approx 80 \mathrm{~nm}$. The strong overall dipole moment (including RT sidebands) is responsible for a spontaneous emission rate of $0.04 \mathrm{GHz}$ at room temperature. At lower temperatures $(\sim 2 \mathrm{~K})$, the ZPL prevails at $638 \mathrm{~nm}$ with a spontaneous emission of $0.013 \mathrm{GHz}$. NV centers are interesting due to their stability, homogeneous properties, and long spin coherence times, making them ideal for quantum information processing tasks, as has been recently reviewed in Ref. 64.

The second emitter we investigate are chemically synthesized CdSe semiconductor quantum dots. These spherical nanocrystals with diameters of the order of several nanometers can also be operated at RT and show a sizetunable emission wavelength in the red part of the spectrum. A typical example is a quantum dot with a spectral width $\approx 20 \mathrm{~nm}$ at $650 \mathrm{~nm}$ (Refs. 65 and 66) and spontaneous emission rate of $0.05 \mathrm{GHz}$ at room temperature. ${ }^{66}$ Finally, we study quantum dots made out of InAs clusters in GaAs. ${ }^{67,68}$ They can exhibit very strong dipole moments and spontaneous emission rates above $1 \mathrm{GHz}$ at cryogenic temperatures $(T \sim 4 \mathrm{~K})$.

\section{COUPLING}

\section{A. Strong coupling condition}

The eigenvalues of Eq. (14) form the so-called JC ladder. At resonance $\omega_{r}=\omega_{e}$, the states split in doublets $\left|\psi_{ \pm}\right\rangle=$ $1 / \sqrt{2}(|N, g\rangle \pm|N-1, e\rangle)$ with energies $E_{n, \pm}=\hbar N \omega_{0} \pm$ $\hbar \sqrt{N} g$. The degeneracy between TLS and resonator is lifted because of the coupling, yielding an anticrossing splitting of $2 g \sqrt{N}$. Considering the smallest level repulsion with one photon $N=1$, the SC condition is usually settled as the parameter range where the emission spectrum of the cavity emitter consists on two peaks of different frequencies. ${ }^{54}$ This is the case if ${ }^{54,69,70}$

$$
|g|>\frac{1}{4}\left|\gamma_{r}-\gamma_{e}\right|
$$

Here, we neglected emitter dephasing. In the opposite case, the losses dominate over the coupling and a splitting of the lines can not be resolved. This is the $\mathrm{WC}$ regime.

Reaching SC is the objective in many cavity QED experiments. From the fundamental point of view, resolving the $\left|\psi_{ \pm}\right\rangle$states confirms the quantum nature of the light-matter coupling. Being in the SC regime has multiple practical applications as well, which we will discuss to some extent in the next section for the case of plasmons. Nevertheless, the WC regime has its own interest, e.g., for effective single-photon generation. In both cases, the ratio of coupling over losses should be as large as possible. In the following, we compute the coupling and losses in the case of different plasmonic resonators. The number of parameters to play is huge, so a brute force exploration is unpractical. Therefore, we first look at the dependencies of both the coupling and losses on different parameters, which will help us to find the most promising configurations for achieving strong coupling.

From Eq. (13) we see that $\left.g \sim \sqrt{\Gamma_{0} /\left(A_{\text {eff }} L\right.}\right)$. The coupling strength depends on the emitter via its free-space spontaneous emission and, therefore, its dipole moment $\Gamma_{0} \sim|d|^{2}$. Larger dipole moments directly translate to higher couplings. The two remaining dependencies come from the resonator itself. The first one is the transverse field confinement $\sim 1 / A_{\text {eff }}$. The very small modal area of plasmons was the motivation to investigate plasmon resonators in the first place. Finally, the coupling depends on the field strength at the emitter position and consequently $g \sim 1 / \sqrt{L}$.

Now, we turn to the right side of the SC condition [Eq. (17)], quantifying decoherence of emitter and resonator. To minimize resonator losses in Eq. (11) we must search for long propagation lengths and highly reflective mirrors. Besides, we see an interesting dependence with $1 / L$ in $\gamma_{\text {leak }}$ [cf. Eq. (11) and discussion below]. This must be compared to the $1 / \sqrt{L}$ dependence of the coupling strength. Therefore, in a realistic scenario where the reflectivity is always less than one, these two dependencies compete and, depending on the other parameters, an optimal length appears. This discussion is also 
true for the ring configuration by replacing leakage through the mirrors by bending losses. The latter also decreases when increasing the resonator length (but this time exponentially ${ }^{59}$ ) since the curvature is reduced. Therefore, pretty much like in the linear case, for circular resonators an optimal length also appears.

\section{B. Temperature and propagation losses}

In the next section, we will see that the losses from the plasmon resonators are often dominated by the small propagation length of the plasmons. In particular, it may not be sufficient to use sophisticated waveguide geometries to increase this length. An often overlooked factor affecting plasmon propagation length is temperature since usual plasmonic experiments are operated at room temperature. However, lowering the temperature, the propagation length of plasmons can be systematically extended by orders of magnitude. ${ }^{26}$ This increase is possible if the conduction losses inside the metal are dominated by scattering by phonons instead of defects such as grain boundaries or impurities. Furthermore, the metal nanostructure must have a smooth surface or otherwise electron scattering at the surface will dominate $^{15,71}$ (as well as radiative losses but they are much smaller ${ }^{15}$ ).

Using the Drude-Sommerfeld model for free electrons, the imaginary part of the permittivity $\epsilon^{\prime \prime}$ is approximately proportional to the resistivity $\rho$ (details in Appendix D). Since $\left|\epsilon^{\prime}\right| \gg\left|\epsilon^{\prime \prime}\right|$, the modal properties of the plasmons are not affected and a decrease in resistivity directly translates into an increase in propagation length

$$
\ell \propto \frac{1}{\epsilon^{\prime \prime}} \propto \frac{1}{\rho(T)} .
$$

Working at lower temperatures is of course an experimental hurdle. However, many quantum emitters must be operated at low temperatures anyway. In sufficiently smooth, pure, and single-crystalline silver, the propagation length can be easily enhanced by a factor of about 10 when using liquid nitrogen (77 K) or even almost 100 when using liquid helium (4 K) (see Appendix D).

\section{Strong and weak coupling in plasmon resonators}

Now, we present a systematic study on whether the SC condition (17) can be fulfilled with combinations of realistic plasmon waveguides, resonator geometries, and emitters. Since this depends on so many adjustable parameters (different waveguides each with different geometries, resonator reflectivity and length, temperature, emitters) it is convenient to have a representation that gives a broad overview for as many of these parameters as possible. To this end, we rearrange the SC condition [Eq. (17)] as

$$
\sqrt{\frac{3}{m} \frac{\Gamma_{0}}{\omega} \frac{v_{p} c}{v_{g}^{2}} \frac{A_{0}}{A_{\mathrm{eff}}}}>\frac{1}{8}\left(\frac{\ell}{\lambda}\right)^{-1}-\frac{1}{2 m} \ln |R| .
$$

We have neglected the emitter losses into nonplasmonic modes and used the relation $\omega=v_{p} k^{\prime}$. Notice that the properties of the waveguide are encoded in only two parameters: (i) the field confinement $\frac{v_{p} c}{v_{g}^{2}} \frac{A_{0}}{A_{\text {eff }}}$ and (ii) the propagation length normalized

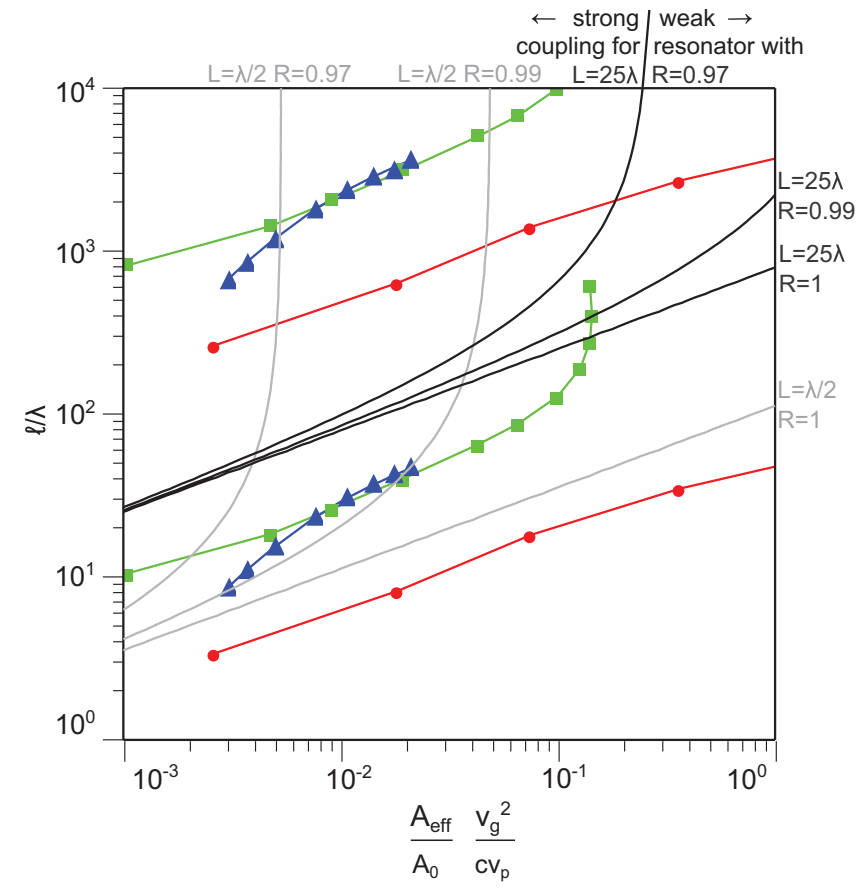

FIG. 4. (Color online) Overview of conditions for reaching SC for different resonators, made from wedge (blue triangles), hybrid (green squares), and nanowire (red dots) waveguides. The emitter is assumed to have $\lambda_{0}=1550 \mathrm{~nm}$ and $\Gamma_{0}=1 \mathrm{GHz}$. We draw lines separating the region of strong and weak coupling for multiple resonator realizations with different lengths $(L=\{\lambda / 2,25 \lambda\})$ and end reflectivities $(R=\{1,0.99,0.97\})$. The considered waveguides are marked by symbols and are defined by the following parameters (the order in the parameter list corresponds to the order on the waveguide line counted from the leftmost point). The nanowire waveguide radii are $r_{\text {rad }}=\{25,50,100,250,500,750,1000\} \mathrm{nm}$. The wedge waveguide angles are $\theta=\{5,10,20,40,60,80,100,120\}^{\circ}$ and its tip has a radius of $10 \mathrm{~nm}$. The hybrid waveguide has separations between the metal and the dielectric nanowire of $h=$ $\{5,25,50,100,200,300,500,750,1000,1250,1500\} \mathrm{nm}$ and its dielectric nanowire a width of $200 \mathrm{~nm}$. Furthermore, we show results for waveguides both at room temperature (lower three lines) and $T=4 \mathrm{~K}$ (upper three lines).

to the plasmon wavelength $\ell / \lambda$. By choosing them to be the axes of a $2 \mathrm{D}$ plot $^{16}$ in Fig. 4, we can represent the boundaries that separate strong and weak coupling regimes, which are independent of the actual waveguide used. Furthermore, this can be done for various resonator lengths $[L=m \lambda / 2$, see Eq. (10)] and mirror reflectivities $(|R|)$. Notice that the lines with $R=1$ in Fig. 4 can also represent circular resonators which are long enough to have negligible bending losses (for instance, the line with $\{L=25 \lambda, R=1\}$ ).

We can now overlay in this figure the achievable field confinements and propagation lengths for different waveguides (nanowire, hybrid, wedge) as function of the geometrical parameters that define them. Within this representation, an emitter and a resonator made of a particular waveguide are in the SC regime if the point corresponding to the waveguide is above (meaning higher propagation length than the minimum required) and to the left (meaning higher confinement than needed) of the relevant given boundary line. 
The calculations of propagation lengths and field confinements were carried out numerically via a finite-element method. The emitter is placed at $10 \mathrm{~nm}$ from the metal surface. In this way, as mentioned above, the coupling into nonradiative channels is strongly suppressed while the coupling into plasmons is as large as if the emitter were at the surface. Beyond this restriction, both emitter position and orientation were optimized to provide maximal coupling into surface plasmons.

Figure 4 considers an emitter operating at telecom frequencies (free-space wavelength $\lambda_{0}=1550 \mathrm{~nm}$ ), for instance, a self-assembled InAs/GaAs quantum dot. As we can see, SC is hard to achieve at room temperature. With nonperfectly reflecting mirrors $(R=0.99)$, only the hybrid waveguide can lead to resonators in the $\mathrm{SC}$ region. The emitted and resonators made of wire or wedge waveguides are always in WC. The propagation lengths computed for $T=4 \mathrm{~K}$ are two orders of magnitude larger than those computed assuming room temperature. Of course, imperfections in the waveguides may limit such enhancements in propagation length. However, our calculations show that even at the lower propagation lengths, the improvement when lowering the temperature places the system comfortably in the SC phase space, especially for long resonators with good reflective ends.

We can conclude that strong coupling should be reachable at low temperatures between a single high-dipole moment quantum dot (e.g., InAs/GaAs) and a plasmonic resonator. This is possible by using (chemically synthesized) smooth singlecrystalline waveguides, realistic DBR mirror reflectivities above $95 \%$, and resonator lengths of several wavelengths.

\section{NV center or CdSe QDs}

In Fig. 5 we plot the same as in Fig. 4 but for emitters with spontaneous emission rate of $\Gamma_{0}=0.05 \mathrm{GHz}$ at $\lambda_{0}=$ $650 \mathrm{~nm}$. This resembles optimistic values for CdSe QDs or $\mathrm{NV}$ centers. In this case, the SC regime is harder to reach: the emission rate is smaller and the normalized propagation length of most of the waveguides is shorter at optical than at telecom frequencies. Even at lowered temperatures, reaching SC with emitters with such low emission rates presents an experimental challenge. Especially, since at optical frequency interband transitions, which are independent of temperature, limit the propagation-length increase that can be achieved when lowering the temperature.

\section{E. Tradeoff between confinement and losses}

In Fig. 4 we first notice the well-known tradeoff between mode confinement and propagation length for plasmon waveguides: ${ }^{72}$ the parametric lines for each waveguide run more or less diagonal from bottom-left to top-right in the 2D parameter-space plots. However, different waveguide types perform differently, with the hybrid waveguide offering highest field confinement and propagation lengths, as noted in Ref. 16. The mode confinement in the plane perpendicular to the waveguide axis therefore affects the maximum achievable quality factor of the resonator.

Interestingly, we see that the trend observed for plasmon waveguides also holds for the resonators as the stronger the

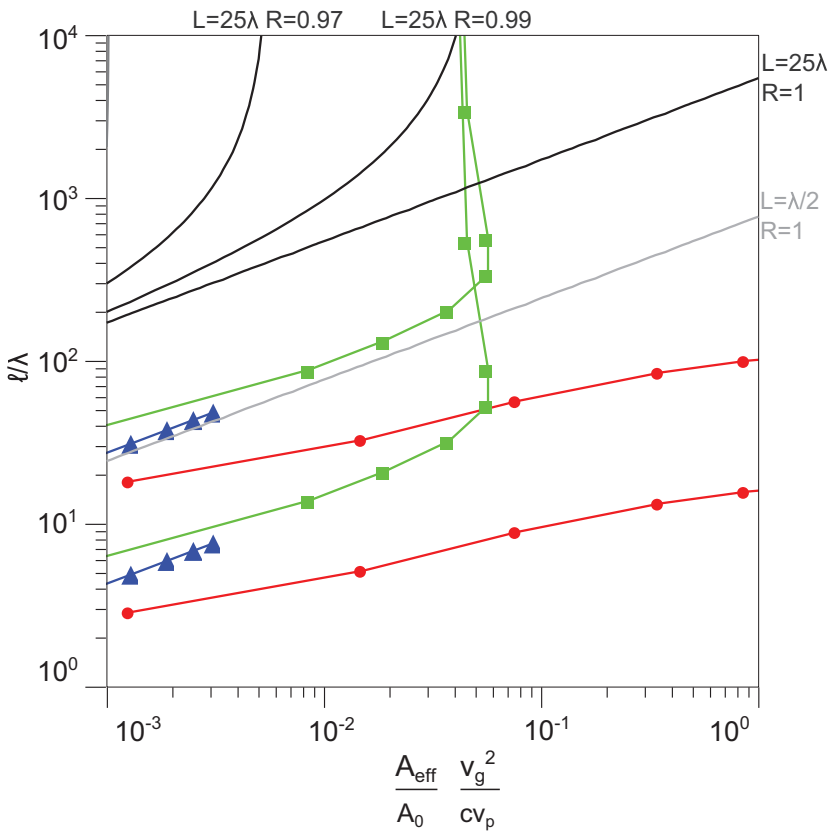

FIG. 5. (Color online) Same plot as in Fig. 4 but with an emitter operating at $\lambda_{0}=650 \mathrm{~nm}$ and a free-space spontaneous emission rate of $\Gamma_{0}=0.05 \mathrm{GHz}$. This roughly corresponds to CdSe QDs or NV centers. The waveguide properties are all the same as in Fig. 4 except the dielectric nanowire that has been adapted to better perform at this wavelength with a width of $100 \mathrm{~nm}$.

field confinement in the dimension along the waveguide (e.g., shorter resonators), the higher the losses at the ends of the resonators. This is also true for circular resonators, where building shorter but more strongly bent resonators results in higher bending losses.

\section{APPLICATIONS}

Let us discuss some practical applications of the theory presented so far.

\section{A. Purcell enhancement for single-plasmon sources}

As derived in Appendix C, the Purcell factor for plasmon resonators, i.e., emission into surface plasmons compared to the emission if the emitter would be placed in a homogeneous dielectric with $\epsilon_{d}$, is

$$
F=F_{\text {guide }} \times F_{\text {res }}=\frac{3}{\pi \epsilon_{d}^{3 / 2}} \frac{c}{v_{g}} \frac{A_{0}}{A_{\text {eff }}} \times \frac{4}{m \pi} \frac{v_{g}}{v_{p}} \frac{1}{\frac{1}{Q_{d}}+\frac{1}{Q_{r}}} .
$$

The first factor is a broadband enhancement due to the strong transversal field confinement $\left(\propto 1 / A_{\text {eff }}\right)$ and reduced group velocity for propagation of EM fields $\left(\propto 1 / v_{g}\right)$ in plasmonic waveguides. The second part is a resonant (wavelengthdependent) enhancement arising from the longitudinal confinement in the resonator.

At room temperature, the solid-state emitters presented in Sec. IIIC exhibit broad bandwidths due to dephasing. This can be efficiently expressed in terms of the quality factor of the emitter $Q=\lambda_{0} / \Delta \lambda$, where $\Delta \lambda$ is the linewidth 


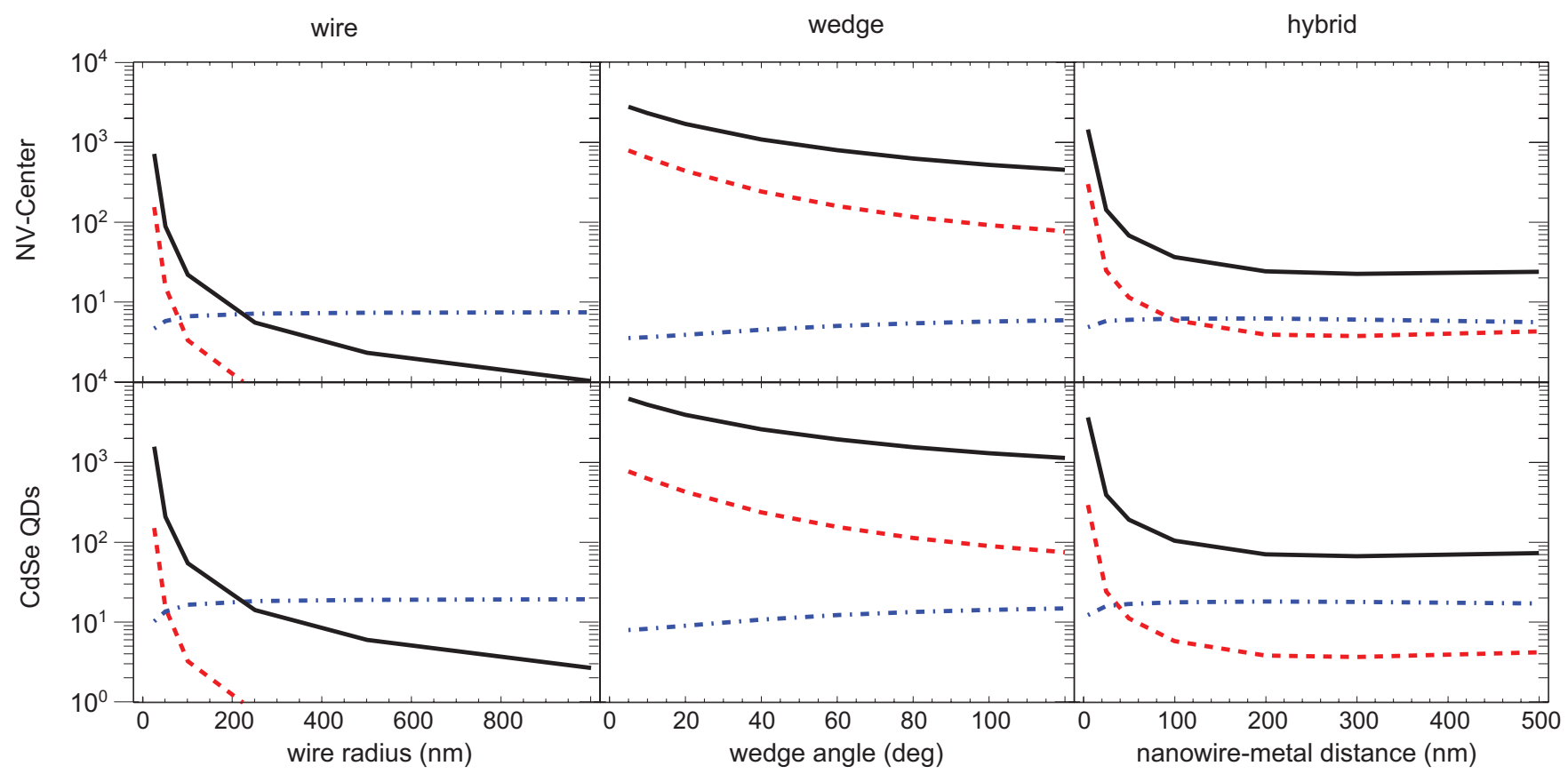

FIG. 6. (Color online) Purcell factors for plasmon resonators made of different waveguides. The emitters used are NV centers and CdSe QDs at room temperature with a a free-space wavelength of approximately $650 \mathrm{~nm}$. The reflectivity of the resonator ends is $|R|=0.97$ and its length $L=\lambda / 2$. The red dashed line is the Purcell factor due to transverse mode confinement of the guided modes ( $\left.F_{\text {guide }}\right)$. The blue dashed-dotted line is the Purcell factor originating from the resonator $\left(F_{\text {res }}\right)$. The solid black line is the total Purcell factor. For higher confined modes (which, for each panel, occur at small values in the abscissa), the resonator Purcell factor decreases since the higher losses decrease the quality factor of the resonator. For weakly confined long-propagating modes (large values in the abscissa), the resonator Purcell factor is limited by the emitter's quality factor.

of the emitter almost entirely attributed to dephasing. For popular emitters such as NV centers and CdSe quantum dots, the quality factors are $Q_{d, \mathrm{NV}} \approx 670 \mathrm{~nm} / 80 \mathrm{~nm} \approx 8$ and $Q_{d, \mathrm{CdSe}} \approx 650 \mathrm{~nm} / 20 \mathrm{~nm} \approx 33$, respectively. ${ }^{64,65}$ These values are considerably smaller than those for typical plasmon resonators and therefore limit the achievable Purcell factors. This can be understood if we visualize that the linewidth of the resonator is much smaller than that of the emitter. Therefore, the resonator EM modes only resonate with a small part of the emitter emission spectrum. The advantage of using plasmon resonators is that the subwavelength field confinement related to the underlaying waveguide allows a very high broadband enhancement of the coupling to the emitter. ${ }^{8,73,74}$ Indeed, as we can see in Fig. 6, the Purcell factor due to transversal mode confinement is responsible for very high overall Purcell factors. This is even true for very broad emitters at room temperature where Purcell factors above 1000 are possible. We especially see that the decrease in propagation length of more confined modes does not play the dominant role here: the enhancement of the broadband Purcell factor due to mode confinement amply exceeds the decrease of the resonant Purcell factor due to the reduction in propagation length.

Let us point out that there are experimental realizations where our theory can be applied. In a recent experiment, ${ }^{8}$ Purcell factors of 75 have been reported for CdSe QDs when coupled to a 50-nm-radius nanowire embedded in PMMA. Taking the experimental reported parameters for $L=\lambda$ and $\mathrm{DBR}$ mirror reflectivity $\approx 0.95$, we calculate a Purcell factor of 64 for a distance between the QD and the wire surface of
$10 \mathrm{~nm}$. This is a very satisfactory agreement with the reported value, especially when taking into account that our calculations do not involve any fitting parameter. Furthermore, a look at lowered temperatures is also interesting here. Higher Purcell factors at lower temperatures can be expected due to higherquality factors of both resonators and emitters.

Although, in the considered cases, the resonant contribution to the Purcell factor $\left(F_{\text {res }}\right)$ is smaller than the broadband contribution due to the coupling to the strongly confined waveguide $\left(F_{\text {guide }}\right)$, it has several features that are important for single-plasmon sources. First of all, $F_{\text {res }}$ still has a value above 5 for the broadband emitters considered in Fig. 6 and therefore it is a significant contribution to the high total Purcell factor. Second, it selectively enhances plasmons with large propagation lengths since it is an effect attributed to cavity resonance. Only the SPP which match the cavity length are enhanced. This is particularly important when dealing with the "efficiency" to emit radiation into this SPP rather than into other SPPs or nonradiative modes.

\section{B. Strong coupling}

Once we know under which conditions the SC regime is reachable within plasmonic resonators, we go through some applications. As anticipated in the Introduction, cavity QED systems in the strong coupling regime are a cornerstone in quantum optics and a huge number of applications were proposed and implemented in different realizations. Let us discuss some of them that may have relevance in the 


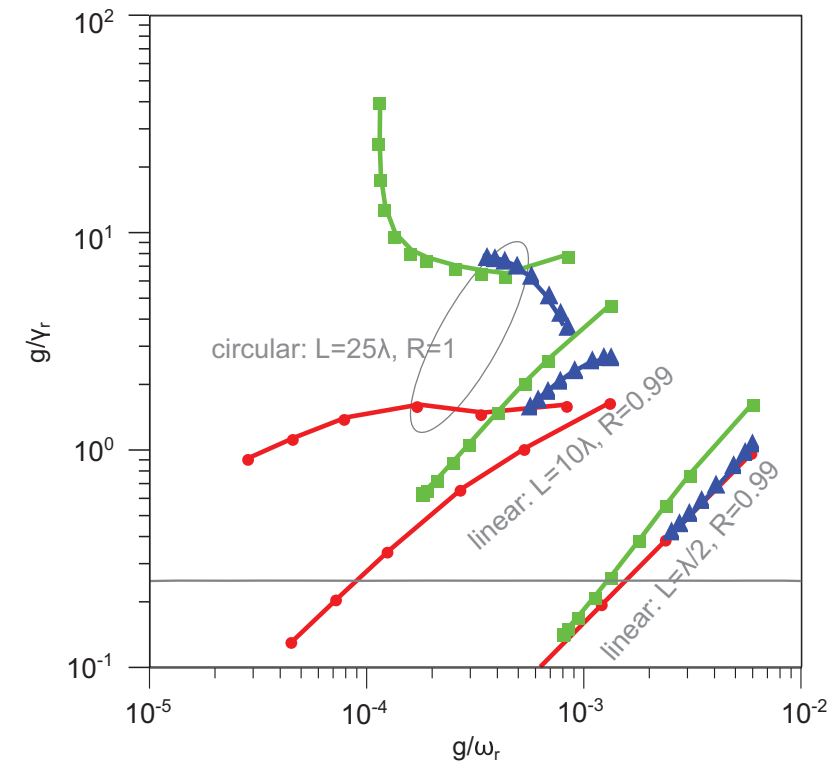

FIG. 7. (Color online) Coupling strength over losses for an $\Gamma_{0}=1 \mathrm{GHz}$ emitter at $\lambda_{0}=1550 \mathrm{~nm}$ at $4 \mathrm{~K}$ for different resonator realizations. The underlying waveguide parameters are varied as in Fig. 4. The considered waveguides are marked by symbols and are defined by the following parameters (the order in the parameter list corresponds to the order on the waveguide line counted from the leftmost point). The nanowire waveguide radii are $r_{\text {rad }}=\{25,50,100,250,500,750,1000\} \mathrm{nm}$. The wedge waveguide angles are $\theta=\{5,10,20,40,60,80,100,120\}^{\circ}$ and its tip has a radius of $10 \mathrm{~nm}$. The hybrid waveguide has separations between the metal and the dielectric nanowire of $h=$ $\{5,25,50,100,200,300,500,750,1000,1250,1500\} \mathrm{nm}$ and its dielectric nanowire a width of $200 \mathrm{~nm}$. Above the gray line, the strong coupling condition is fulfilled.

manipulation of light at the nanoscale. All those applications are implicitly or explicitly related to the coherent coupling between the TLS and the resonator mode, encapsulated in the ratio $g / \omega_{r}$ and $g / \max \left[\gamma_{r}, \gamma_{e}, \gamma_{d}\right]$. The larger the coupling and smaller the losses, the faster and more coherent light-matter oscillations are, optimizing the performance of many of the applications. For later reference, we plot in Fig. 7 the expected performance of $g / \gamma_{r}$ as a function of $g / \omega_{r}$ for several plasmonic resonators operated at $4 \mathrm{~K}$, together with the boundary separating the strong and weak coupling regimes. We consider linear resonators with different lengths and a mirror reflectivity $|R|=0.99$ but, for the larger resonator length considered, the results are also applicable to circular resonators, as in this case bending losses are expected to be negligible. For ease of comparison to other coupled lightmatter systems, Fig. 8 reproduces the results rendered in Fig. 7 , but in terms of explicit values for the coupling and the loss rates.

a. Quantum nonlinear optics. The JC model (14) is nonlinear, the energy levels are not equally spaced. Therefore, its response to an external stimulus is not linear either. In the dispersive regime, ${ }^{75}$ by expanding the $\mathrm{JC}$ model in powers of $g / \delta \ll 1$ with $\delta=\omega_{r}-\omega_{e}$, effective Kerr Hamiltonians such as $H / \hbar=\omega a^{\dagger} a+\kappa\left(a^{\dagger} a\right)^{2}$ have been proposed. ${ }^{76}$ Kerr nonlinearities generate squeezed states. In a circuit-QED

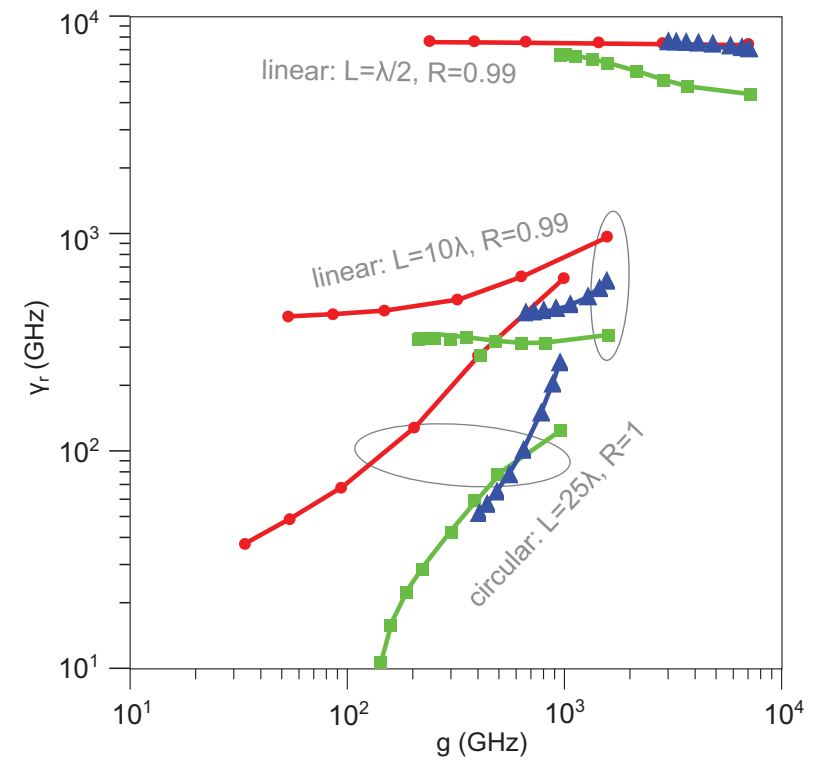

FIG. 8. (Color online) Absolute values of coupling and resonator loss rates for the same cases considered in Fig. 7.

implementation, such physics has been recently reported. ${ }^{10}$ In that work, the authors exploited these nonlinearities to demonstrate, among other things, squeezing. In this experiment, $g / \gamma_{e} \sim 10$ and $g / \omega \sim 10^{-3}$. As shown in Fig. 7, these numbers can be reproduced with the plasmonic resonators considered in this paper.

b. Plasmon-plasmon interaction. Rooted in the same nonlinearities, the JC physics can be used to induce effective photon-photon interactions, as in the so-called photon blockade phenomenon. ${ }^{77}$ Another evidence for photon-photon interactions in cavity QED systems has been demonstrated in Ref. 11, where two light beams interact through a QD (InAs) coupled to a cavity in a photonic crystal. In that experiment, the reporting numbers are $g / \gamma_{r} \sim 1$ and $g / \omega \sim 10^{-4}$. Again, these numbers are within reach of plasmonic resonators (see Fig. 7).

c. Other. Photon-photon interaction allows exploring BoseHubbard-type models in JC lattices, i.e., arrays of coupled cavity-TLS systems. The nonequally spaced levels in the JC model can yield two phases (localization and delocalization) depending on the coupling $g$ and the hopping term between the cavities. ${ }^{78}$ These phases survive even the presence of dissipation. ${ }^{79}$ Cavity QED is also a building block for quantum information tasks. On the other hand, demonstrations of quantum computation, ${ }^{80}$ state tomography, ${ }^{81}$ or quantum buses ${ }^{82}$ were done in systems exceeding the ratios $g / \max \left[\gamma_{r}, \gamma_{e}, \gamma_{d}\right]$ presented here. This may be a motivation to further improve current figures, for instance, the mirror reflectivities, in order to enable these tasks in plasmonic resonators. Finally, we mention recent advances in doing quantum physics driven by dissipation. ${ }^{83,84}$ There, dissipation is viewed as beneficial for reaching interesting ground states or doing quantum computation. Because strong dissipation is present in quantum plasmonics, further investigation in this direction seems rewarding. 


\section{CONCLUSIONS}

We have reported a quantum theory for plasmonic resonators coupled to single quantum emitters. Starting from a first-principles theory, and taking into account the main losses, we were able to end up in a master equation for the effective JC model [cf. Eq. (15)]. All the coefficients can be obtained via the classical Green's function together with the emitter characteristics. This allows us to profit from the studies on plasmonic waveguides. We have studied different architectures for optimizing the binomia of field enhancement and losses to reach the SC regime. We have numerically demonstrated that SC in plasmonics QED is possible at cryogenic temperatures. Albeit it is demanding at room temperature, it is possible if further improvements are reached, e.g., higher mirror reflectivities. Importantly enough, our calculations agree with recent experimental results in the WC regime with plasmonic resonators made of nanowires (see our Sec. V A). Furthermore, we have shown that other architectures, such as hybrid or wedge waveguides, can overcome the nanowire implementation and reach even higher Purcell factors. In the paper we also compare the capabilities of plasmonic resonators with other technologies. As demonstrated, plasmonic QED can be used as an effective Kerr media or for generating plasmon-plasmon interactions, demonstrating its feasibility for controlling the few plasmon dynamics at the nanoscale.

\section{ACKNOWLEDGMENTS}

This work was supported by Spanish MICINN Projects No. FIS2011-25167, No. MAT2011-28581-C02, and No. CSD2007-046-Nanolight.es. F.J.G.-V. acknowledges financial support by the European Research Council, Grant No. 290981 (PLASMONANOQUANTA).

\section{APPENDIX A: PLASMON-EMITTER COUPLING HAMILTONIAN}

In this appendix, we justify Hamiltonian (4) in the main text. First, we quantize the macroscopic Maxwell equations (taking into account the losses in the metal). Then, we provide the emitter-plasmon interaction Hamiltonian. The material presented here summarizes the formalism used in Refs. 29, 30, and 85.

\section{EM quantization in dispersive media: System-environment approach}

Let us sketch the quantization program in dispersive (and therefore also lossy) media. We follow an open system approach where the losses are modeled by a reservoir (or "bath") accounting for the irreversible leakage of energy from the system. We find a quantum Langevin equation, which in the classical limit is the Maxwell equation of the EM fields. ${ }^{29,30}$

It is convenient to work both in reciprocal space

$$
\vec{E}(\vec{r}, t)=\frac{1}{(2 \pi)^{3 / 2}} \int d^{3} k \vec{E}(\vec{k}, t) e^{i \vec{k} \cdot \vec{r}},
$$

and in the Coulomb gauge

$$
\vec{A} \cdot \vec{k}=0 \text {. }
$$

The quantization is based on a system-bath Lagrangian. The EM field is coupled to an infinite collection of resonator. The latter provide linear dissipation. Being specific, we write $L_{\text {total }}=\int d^{3} k \mathcal{L}_{\text {total }}$ with

$$
\begin{aligned}
\mathcal{L}_{\text {total }}= & \epsilon_{0}\left(|\dot{\vec{A}}|^{2}-c \vec{k}^{2}|\vec{A}|^{2}\right)+\sum_{j}\left(\mu \dot{x}_{j}^{2}-\omega_{j}^{2} x_{j}^{2}\right) \\
& +\sum_{j} \alpha_{j} \int d^{3} k\left(\dot{\vec{x}}_{j} \vec{A}^{*}+\dot{\vec{x}}_{j}^{*} \vec{A}\right) .
\end{aligned}
$$

The first line accounts for the EM Lagrangian both in the reciprocal space and Coulomb gauge and the set of oscillators. The second line accounts for the interaction. To alleviate the notation, we omit the explicit dependence on $\vec{k}$ and $t$ in $\vec{A}(\vec{k}, t)$. The introduced constants will later on be identified with the system's material parameters.

With Lagrangian (A3) at hand, we start the quantization of $\vec{A}(\vec{k}, t)$ and their conjugate momenta

$$
\Pi=\frac{\partial \mathcal{L}}{\partial \dot{\vec{A}}^{*}}, \quad \Pi^{*}=\frac{\partial \mathcal{L}}{\partial \dot{\vec{A}}} .
$$

The quantized fields satisfy the commutation relations ${ }^{48}$

$$
\left[\vec{A}(\vec{k}), \vec{\Pi}\left(\vec{k}^{\prime}\right)\right]=0, \quad\left[\vec{A}(\vec{k}), \vec{\Pi}^{\dagger}\left(\vec{k}^{\prime}\right)\right]=i \hbar \delta\left(\vec{k}-\vec{k}^{\prime}\right)
$$

and the bath's coordinates satisfy

$$
\left[\vec{x}_{j}, \vec{p}_{j^{\prime}}\right]=i \hbar \vec{\delta}_{j j^{\prime}}
$$

We write the Heisenberg equations of motion for both $\mathbf{A}$ and the bath operators $x_{j}$. Because of the interaction part [cf. third term in (A3)], these equations are coupled

$$
\begin{gathered}
\epsilon_{0} \ddot{\vec{A}}=-c^{2} \vec{k}^{2} \vec{A}-\sum_{j} \alpha_{j} \vec{x}_{j}, \\
\ddot{\vec{x}}_{j}=-\omega_{j}^{2} \vec{x}_{j}+\frac{\alpha_{j}}{\mu} \dot{\vec{A}} .
\end{gathered}
$$

The solution of (A8) is given by

$$
\dot{\vec{x}}_{j}=i \sqrt{\frac{\hbar \omega_{j}}{2 \mu}}\left(f_{j}^{\dagger} e^{i \omega_{j} t}-f_{j} e^{-i \omega_{j} t}\right)-\frac{\alpha_{j}}{\mu \omega_{j}} \int_{-\infty}^{t} \sin \left[\omega_{j}\left(t-t^{\prime}\right)\right] \ddot{\vec{A}}
$$

with the annihilation/creation operators: $\left[f_{i}, f_{j}^{\dagger}\right]=\delta_{i j}$. Inserting the above (A9) in (A7) together with some algebra we end up with an equation for the Fourier components of the vector potential

$$
\vec{A}(\vec{k}, t)=\int d \omega e^{-i \omega t} \vec{A}_{\omega}(\vec{k})
$$

that can be cast to a Langevin-type form

$$
\begin{aligned}
-\epsilon(\omega) \omega^{2} \vec{A}_{\omega}= & -c^{2} \vec{k}^{2} \vec{A}_{\omega}-i \sqrt{\frac{\hbar}{\pi}} \int d \nu v \sqrt{\epsilon^{\prime \prime}(\nu)} \\
& \times\left(f_{\nu}^{\dagger} e^{i v t}-f_{\nu} e^{-i v t}\right)
\end{aligned}
$$

The introduced permittivity of the media

$$
\begin{aligned}
\epsilon(\omega) & =\epsilon^{\prime}(\omega)+i \epsilon^{\prime \prime}(\omega) \\
& =1+\frac{1}{\epsilon_{0}}\left(\mathcal{P}\left[\int d \nu \frac{J(\nu)}{\nu-\omega}\right]+i \frac{\pi}{2} J(\omega)\right) .
\end{aligned}
$$


Notice that we have introduced the imaginary part $\epsilon^{\prime \prime}(\omega)$ also appearing in the integrand of (A11) and the spectral density

$$
J(\omega)=\sum_{j} \frac{\alpha_{j}^{2}}{\mu \omega_{j}} \delta\left(\omega-\omega_{j}\right) .
$$

Equations (A12) and (A13) link the macroscopic complex permittivity function with a microscopic model accounting for linear dissipation. For example, in the Drude-Sommerfeld model [Eq. (D1)], we can write that for frequencies close to the plasma frequency the relation becomes

$$
J(\omega) \cong \epsilon_{0} \frac{\pi}{2} \frac{\Gamma_{\mathrm{el}}}{\omega_{p}^{2}} \omega
$$

with $\omega_{p}$ the plasma frequency and $\Gamma_{\mathrm{el}}$ is the damping parameter. Finally, and understanding the last term in (A11) as the source, rewriting in positionlike operators as $f(\vec{r}, \omega)=$ $1 /(2 \pi)^{3 / 2} \int d^{2} k f(\vec{k}, t)$, we express the fields via the Green's function as expressed in Eq. (1).

\section{Emitter-plasmon coupling: Some formulas}

As argued in the main text, the emitter-plasmons coupling in the dipole-dipole approximation is given by

$$
H_{\text {int }}=-\sigma_{x} \vec{d} \cdot \vec{E}\left(\vec{r}_{e}\right)
$$

with $\vec{E}\left(\vec{r}_{e}\right)=\int_{0}^{\infty} d \omega \vec{E}\left(\vec{r}_{e}, \omega\right)$. Using (1) we can express this interaction Hamiltonian as

$$
H_{\mathrm{int}}=-\sigma_{x} \int_{0}^{\infty} d \omega \int d^{3} r^{\prime} g\left(\omega, r^{\prime}, r_{e}\right)\left[f^{\dagger}\left(\vec{r}^{\prime}, \omega\right)-f\left(\vec{r}^{\prime}, \omega\right)\right] \text {, }
$$

where we have introduced the shorthand notation

$$
g\left(\omega, r^{\prime}, r_{e}\right)=i \sqrt{\frac{\hbar}{\pi \epsilon_{0}}} \frac{\omega^{2}}{c^{2}} \sqrt{\epsilon^{\prime \prime}\left(\vec{r}^{\prime}, \omega\right)} \vec{d} \stackrel{\leftrightarrow}{G}\left(\vec{r}_{e}, \vec{r}^{\prime}, \omega\right) .
$$

We now define the collective modes $a(\omega)$ :

$$
\int d^{3} r^{\prime} g\left(\omega, r^{\prime}, r_{e}\right) f\left(r^{\prime}, \omega\right) \equiv \hbar g(\omega) a(\omega)
$$

which fulfill the bosonic commutation relations

$$
\left[a(\omega), a^{\dagger}\left(\omega^{\prime}\right)\right]=\delta\left(\omega-\omega^{\prime}\right)
$$

and yield

$$
\begin{aligned}
|g(\omega)|^{2}= & \frac{1}{\hbar \pi \epsilon_{0}} \frac{\omega^{4}}{c^{4}} \int d^{3} r^{\prime} \epsilon^{\prime \prime}\left(\vec{r}^{\prime}, \omega\right) \vec{d}^{T} \stackrel{\leftrightarrow}{G}\left(\vec{r}_{e}, \vec{r}^{\prime}, \omega\right) \\
& \times \stackrel{\leftrightarrow}{*}\left(\vec{r}_{e}, \vec{r}^{\prime}, \omega\right) \vec{d} .
\end{aligned}
$$

Using the relation for the Green's tensor ${ }^{31,36}$

$$
\begin{aligned}
& \frac{\omega^{2}}{c^{2}} \int d^{3} r^{\prime} \epsilon^{\prime \prime}\left(\vec{r}^{\prime}, \omega\right) \stackrel{\leftrightarrow}{G}\left(\vec{r}_{e}, \vec{r}^{\prime}, \omega\right) \stackrel{\leftrightarrow}{G^{*}}\left(\vec{r}_{e}, \vec{r}^{\prime}, \omega\right) \\
& \quad=\operatorname{Im} G\left(\vec{r}_{e}, \vec{r}^{\prime}, \omega\right),
\end{aligned}
$$

we finally end up with

$$
|g(\omega)|^{2}=\frac{1}{\hbar \pi \epsilon_{0}} \frac{\omega^{2}}{c^{2}} \vec{d}^{T} \operatorname{Im}\left[\stackrel{\leftrightarrow}{G}\left(\omega, \vec{r}_{e}, \vec{r}_{e}\right)\right] \vec{d} .
$$

This expression allows the evaluation of the contribution of different electromagnetic modes to the spectral density.
In our problem, if the emitter is in an intermediate range of distances to the metal surface (larger than $\sim 10 \mathrm{~nm}$ to avoid quenching and smaller than the plasmon confinement in the direction normal to the metal surface), the coupling is mainly into plasmons. For this reason, we separate the SPP modes from the rest, treating explicitly the emitter-plasmon coupling via an interacting Hamiltonian [given by Eq. (4) in the main text]. The coupling between the emitter and the nonplasmonic electromagnetic modes (considered for this problem as "dissipative channels") are described via Lindblad terms, which affect the nonunitary evolution of the density matrix. With this prescription, the operators $a(\omega)$ and $a^{\dagger}\left(\omega^{\prime}\right)$ are now annihilation and creation operators of plasmon modes and, correspondingly, the spectral density is given by Eq. (5) in the main text.

\section{APPENDIX B: GREEN'S FUNCTION OF RESONATORS}

\section{Linear resonator}

To get the Green's function of a resonator, we first assume that the system is translational along the $z$ direction with additional reflections at the resonator ends, effectively reducing the problem to one dimension. We further notice that $G_{1 \mathrm{D}}\left(\omega, z, z^{\prime}\right)$ can be obtained by summing all the waves scattered at the mirrors. A resonator of length $L$ with complex reflection coefficient $R(0 \leqslant|R| \leqslant 1)$ on the resonator ends located at $x_{l}$ and $x_{r}$ therefore yields the 1D Green's function

$$
\begin{aligned}
G_{1 \mathrm{D}}\left(\omega, z, z^{\prime}\right)= & \sum_{n=0}^{\infty}\left(e^{i k 2 L} R^{2}\right)^{n} \frac{i}{2 k}\left(e^{i k\left|z-z^{\prime}\right|}+R e^{i k\left|2 z_{r}-\left(z+z^{\prime}\right)\right|}\right. \\
& \left.+R e^{-i k\left|2 z_{l}-\left(z+z^{\prime}\right)\right|}+R^{2} e^{i k\left(2 L-\left|z-z^{\prime}\right|\right)}\right) .
\end{aligned}
$$

Without loss of generality, we set $z_{r}=L / 2=-z_{l}$.

The coupling of an emitter to the resonator $|g(\omega)|^{2} \propto$ $\operatorname{Im} G_{1 \mathrm{~d}}(\omega, z, z)$, depends on the position $z$. To maximize the coupling, we place the emitter along the resonator axis at an antinode of the electric field.

\section{Circular resonator}

The boundary condition in the circular resonator configuration presents $2 \pi$ periodicity. In a similar way as for the linear case, summing all the different partial waves yields the Green's function

$$
G_{1 \mathrm{D}}\left(\omega, z, z^{\prime}\right)=\sum_{n=0}^{\infty}\left(e^{i k 2}\right)^{n} \frac{i}{2 k}\left(e^{i k\left|z-z^{\prime}\right|}+e^{i k\left(L-\left|z-z^{\prime}\right|\right)}\right) .
$$

Notice that, as expected, in this case the coupling between emitter and resonator does not depend on the emitter position.

\section{Approximating resonances}

The 1D Green's function evaluated at a field antinode and $z=z^{\prime}$ can be written, for both linear and circular resonators, as

$$
G_{1 \mathrm{D}}(\omega, z, z)=\frac{1}{2 k} \frac{i \sinh \theta^{\prime \prime}+\sin \theta^{\prime}}{\cosh \theta^{\prime \prime}-\cos \theta^{\prime}},
$$

where we have defined

$$
\theta=\theta^{\prime}+i \theta^{\prime \prime}=k^{\prime} L+\varphi+i\left(k^{\prime \prime} L-\ln |R|\right)
$$


and $\varphi=\arg (R)$ is the phase picked up by reflection at each resonator end, which is $\approx \pi$ for the linear resonator and zero in the circular configuration,

We see that $\theta^{\prime \prime}$ quantifies the losses, both due to propagation (via the imaginary part of the propagation constant $k^{\prime \prime}$ ), and the losses through the mirrors (via $|R|<1$ ). For radiation losses due to bending in the circular resonator, we can phenomenologically add a term $k_{\text {bend }}^{\prime \prime}$ to the imaginary part of the propagation constant $k^{\prime \prime}$.

The condition for resonances is

$$
L=\frac{\pi}{k_{r}^{\prime}} m=\frac{\lambda}{2} m,
$$

where $m$ is the number of antinodes in the resonator and has to be an even integer for circular resonators and any integer for the linear configuration. In both configurations, the coupling

$$
|g(\omega)|^{2}=\Gamma_{\text {guide }} \frac{1}{\pi} \operatorname{Im}\left\{k G_{1 \mathrm{D}}(\omega, z, z)\right\}
$$

can be approximated by a Lorentzian near a resonance. We approximate the cosine around the center of the resonance peaks

$$
\cos \left(\theta^{\prime}\right) \cong 1-\frac{1}{2} L^{2}\left(k^{\prime}-k_{0}^{\prime}\right)^{2}=1-\frac{L^{2}}{2 v_{g}^{2}}\left(\omega-\omega_{r}\right) .
$$

Therefore, we can write

$$
|g(\omega)|^{2} \cong g^{2} \frac{1}{\pi} \frac{\gamma_{r} / 2}{\left(\omega-\omega_{r}\right)^{2}+\left(\gamma_{r} / 2\right)^{2}}
$$

with the width of the Lorentzian (FWHM) $\gamma_{r}$ being the resonator decay rate

$$
\gamma_{r}=2 \frac{v_{g}}{L} \sqrt{2\left(\cosh \theta^{\prime \prime}-1\right)} \approx 2 \frac{v_{g}}{L} \theta^{\prime \prime}
$$

and the coupling defined as

$$
g=\sqrt{\Gamma_{\text {guide }} \frac{v_{g}}{L}} \sqrt{\frac{\sinh \theta^{\prime \prime}}{\sqrt{2\left(\cosh \theta^{\prime \prime}-1\right)}}} \approx \sqrt{\Gamma_{\text {guide }} \frac{v_{g}}{L}} .
$$

The approximated results were obtained by assuming weak losses $\theta^{\prime \prime} \ll 1$ and, consequently, Taylor expanding $\sinh \left(\theta^{\prime \prime}\right)$ and $\cosh \left(\theta^{\prime \prime}\right)$.

For small enough $\gamma_{r}$ the Lorentzian in Eq. (B7) can be approximated by the expression in Eq. (12), which is used in the mapping to the Jaynes-Cummings model.

\section{APPENDIX C: PURCELL FACTORS}

Applying the Markov approximation, the emitter coupled to a plasmon resonator undergoes exponential decay into surface plasmons, with a rate given by the spectral density at the frequency of the emitter $\omega_{e}$ (Ref. 86) (Fermi's golden rule):

$$
\Gamma_{\text {res }}=2 \pi\left|g\left(\omega_{e}\right)\right|^{2} \text {. }
$$

We define the Purcell factor as the ratio of this emission into surface plasmons compared to $\Gamma_{0}$, the emission if the emitter would be placed in a homogeneous medium characterized by $\epsilon_{d}$ :

$$
F=\frac{\Gamma_{\text {res }}}{\Gamma_{0}} .
$$

If emission into other channels is negligibly small compared to the emission into surface plasmons, this Purcell factor measures the decrease of the emitter lifetime.

In the case of plasmonic resonators, the Purcell factor can be written as the product of two different contributions

$$
F=\frac{\Gamma_{\text {res }}}{\Gamma_{0}}=F_{\text {guide }} \times F_{\text {res }} .
$$

The waveguide Purcell factor $F_{\text {guide }}$ exists even without a cavity. It is a result of the small mode area and higher density of states $\left(\partial k / \partial \omega=v_{g}^{-1}\right)$ of guided surface plasmons

$$
F_{\text {guide }} \equiv \frac{\Gamma_{\text {guide }}}{\Gamma_{0}} \equiv \frac{3}{\pi \epsilon_{d}^{3 / 2}} \frac{c}{v_{g}} \frac{A_{0}}{A_{\text {eff }}} .
$$

Notice that $F_{\text {guide }}$ has no resonance origin, so it is broadband. The additional contribution to the Purcell factor $\left(F_{\text {res }}\right)$ is, for $g \ll \max \left\{\gamma_{r}, \gamma_{p}, \gamma_{e}\right\}$,

$$
F_{\text {res }}=\frac{\Gamma_{\text {res }}}{\Gamma_{\text {guide }}}=\frac{4 g^{2}}{\gamma_{r}+\gamma_{d}} / \Gamma_{\text {guide }},
$$

where $\gamma_{d}$ is the linewidth due to emitter dephasing (spectral diffusion). Using $g^{2}=\frac{v_{g}}{L} \Gamma_{\text {guide }}, \omega=v_{p} 2 \pi / \lambda, L=m \lambda / 2$, $w_{e}=w_{r}$, and the quality factors $Q_{d}=\omega_{e} / \gamma_{d}$ (emitter dephasing) and $Q_{r}=\omega_{r} / \gamma_{r}$ (resonator), we can rewrite the resonator Purcell factor in terms of

$$
F_{\text {res }}=\frac{4}{m \pi} \frac{v_{g}}{v_{p}} \frac{1}{\frac{1}{Q_{p}}+\frac{1}{Q_{r}}} .
$$

The fraction of emission guided into surface plasmons is given by

$$
\beta=\frac{\Gamma_{\text {res }}}{\Gamma_{\text {res }}+\gamma_{e}} .
$$

In normal cavity QED, the emitter decay rates to channels not in the cavity $\left(\gamma_{e}\right)$ stay approximately the same with and without the cavity since the cavity only affects the modes in a small spatial angle. In plasmonics, the presence of the metal surface leaves the emission into radiation modes practically unaltered. However, an absorbing metal surface also introduces decay into nonradiative modes that dissipate in the metal. The decay into nonradiative modes is dominant at distances below $\sim 10-20 \mathrm{~nm}$. Here, even at these distances, the plasmonic cavity is quite useful as it increases the decay into surface plasmons and not the decay into nonradiative modes. This is an advantage of the "resonator Purcell factor" over the "broadband Purcell factor."

\section{APPENDIX D: TEMPERATURE AND PROPAGATION LOSSES}

Since the phonon population is strongly temperature dependent, we can significantly reduce scattering of electrons and increase the plasmon propagation lengths.

To get the permittivity as a function of temperature, we recourse to the Drude-Sommerfeld model for free electrons which is well applicable and sufficient for near-infrared to telecom $\left(\lambda_{0} \approx 1550 \mathrm{~nm}\right)$ wavelengths, where interband transitions can be safely neglected in silver. Here, the permittivity is given by the plasma frequency of the free electrons $\omega_{p}$ and 
the damping rate of the electrons $\Gamma_{\mathrm{el}}$ :

$$
\epsilon_{\text {Drude }}(\omega)=1-\frac{\omega_{p}^{2}}{\omega^{2}+i \Gamma_{\mathrm{el}} \omega} .
$$

The damping is a function of Fermi velocity $v_{F}$ and the meanfree path of the electrons $l_{\mathrm{el}}, \Gamma_{\mathrm{el}}=\frac{v_{F}}{l_{\mathrm{el}}}$. The mean-free path is in turn proportional to the resistivity $\rho, l_{\mathrm{el}} \propto 1 / \rho$. Using $\omega \gg \Gamma_{\mathrm{el}}$, the imaginary part of the permittivity is thus approximately proportional to the resistivity

$$
\epsilon_{\text {Drude }}^{\prime \prime}(\omega) \propto \Gamma_{\text {el }} \propto \rho .
$$

By using tabulated data for the resistivity of silver ${ }^{87}$ at different temperatures and scaling $\Gamma_{\mathrm{el}}$ accordingly, we get the imaginary part of the permittivity at different temperatures. The real part stays approximately constant.

With $\left|\epsilon^{\prime}\right| \gg\left|\epsilon^{\prime \prime}\right|$, the modal shape of the propagating plasmons is not affected and the reduced imaginary part of the permittivity directly translates into increased propagation length. The imaginary part of the permittivity is plotted in Fig. 9. It translates to a propagation-length increase that is universal for all waveguides analyzed in this paper.

At low temperatures, $\epsilon^{\prime \prime}$ saturates since the dominant electron scattering happens at lattice impurities. Furthermore, when the scattering due to the Drude-Sommerfield model vanishes, small but maybe finite interband transitions may play a role. Note that at optical frequencies they may even dominate. That is why the change of $\epsilon^{\prime \prime}$ is less pronounced for

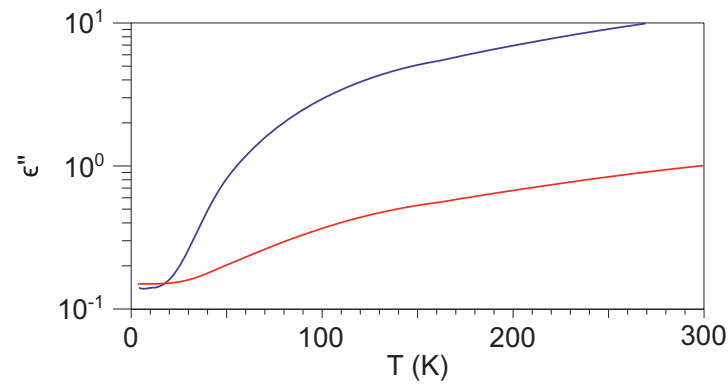

FIG. 9. (Color online) Permittivity of silver as a function of temperature for $\lambda_{0}=1550 \mathrm{~nm}$ (blue line) and $\lambda_{0}=650 \mathrm{~nm}$ (red line). We see that an increase of about 100 is possible in the first case, while an increase of about 10 is possible for optical frequencies.

$\lambda_{0}=650 \mathrm{~nm}$ in Fig. 9. However, the data used for interband transitions ${ }^{39}$ may be very vague at lower temperatures since it is obtained from a fit at room temperature. A more close experimental analysis is needed here.

Of special interest are the properties at 77 and $4 \mathrm{~K}$ reachable with liquid nitrogen and liquid helium, respectively. For $\lambda=1550 \mathrm{~nm}$, the plasmon propagation length at $77 \mathrm{~K}$ is $\sim 5$ times larger than the room-temperature value, while at $4 \mathrm{~K}$ this factor is 80 . Notice that these are estimations obtained from experimental values of the resistivity of silver. Single-crystal resonators may present even larger enhancements.
${ }^{1}$ S. Haroche and J. Raimond, Sci. Am. 54, 26 (1993).

${ }^{2}$ J. Raimond, M. Brune, and S. Haroche, Rev. Mod. Phys. 73, 565 (2001).

${ }^{3}$ X. Zhu, S. Saito, A. Kemp, K. Kakuyanagi, S.-i. Karimoto, H. Nakano, W. J. Munro, Y. Tokura, M. S. Everitt, K. Nemoto, M. Kasu, N. Mizuochi, and K. Semba, Nature (London) 478, 221 (2011).

${ }^{4}$ M. D. LaHaye, J. Suh, P. M. Echternach, K. C. Schwab, and M. L. Roukes, Nature (London) 459, 960 (2009).

${ }^{5}$ G. A. Steele, A. K. Hüttel, B. Witkamp, M. Poot, H. B. Meerwaldt, L. P. Kouwenhoven, and H. S. J. van der Zant, Science 325, 1103 (2009).

${ }^{6}$ K. Hennessy, A. Badolato, M. Winger, D. Gerace, M. Atatüre, S. Gulde, S. Fält, E. Hu, and A. Imamoglu, Nature (London) 445, 896 (2007).

${ }^{7}$ O. Romero-Isart, M. L. Juan, R. Quidant, and J. I. Cirac, New J. Phys. 12, 033015 (2010).

${ }^{8}$ N. P. de Leon, B. J. Shields, C. L. Yu, D. E. Englund, A. V. Akimov, M. D. Lukin, and H. Park, Phys. Rev. Lett. 108, 226803 (2012).

${ }^{9}$ Y. Mu and C. M. Savage, Phys. Rev. A 46, 5944 (1992).

${ }^{10}$ Y. Yin, H. Wang, M. Mariantoni, R. Bialczak, R. Barends, Y. Chen, M. Lenander, E. Lucero, M. Neeley, A. O'Connell, D. Sank, M. Weides, J. Wenner, T. Yamamoto, J. Zhao, A. Cleland, and J. Martinis, Phys. Rev. A 85, 023826 (2012).

${ }^{11}$ D. Englund, A. Majumdar, M. Bajcsy, A. Faraon, P. Petroff, and J. Vučković, Phys. Rev. Lett. 108, 093604 (2012).

${ }^{12}$ T. D. Ladd, F. Jelezko, R. Laflamme, Y. Nakamura, C. Monroe, and J. L. O'Brien, Nature (London) 464, 45 (2010).
${ }^{13}$ T. Niemczyk, F. Deppe, H. Huebl, E. P. Menzel, F. Hocke, M. J. Schwarz, J. J. Garcia-Ripoll, D. Zueco, T. Hümmer, E. Solano, A. Marx, and R. Gross, Nat. Phys. 6, 772 (2010).

${ }^{14}$ P. Forn-Díaz, J. Lisenfeld, D. Marcos, J. García-Ripoll, E. Solano, C. J. P. M. Harmans, and J. E. Mooij, Phys. Rev. Lett. 105, 237001 (2010).

${ }^{15}$ D. E. Chang, A. S. Sørensen, P. R. Hemmer, and M. D. Lukin, Phys. Rev. B 76, 035420 (2007).

${ }^{16}$ R. F. Oulton, G. Bartal, D. F. P. Pile, and X. Zhang, New J. Phys. 10, 105018 (2008).

${ }^{17}$ R. F. Oulton, V. J. Sorger, D. A. Genov, D. F. P. Pile, and X. Zhang, Nat. Photonics 2, 496 (2008).

${ }^{18}$ A. Boltasseva, V. S. Volkov, R. B. Nielsen, E. Moreno, S. G. Rodrigo, and S. I. Bozhevolnyi, Opt. Express 16, 5252 (2008).

${ }^{19}$ S. I. Bozhevolnyi, V. S. Volkov, E. Devaux, J. Laluet, and T. W. Ebbesen, Nature (London) 440, 508 (2006).

${ }^{20}$ M. I. Stockman, Phys. Rev. Lett. 93, 137404 (2004).

${ }^{21}$ V. S. Volkov, S. I. Bozhevolnyi, S. G. Rodrigo, L. Martin-Moreno, F. J. Garcia-Vidal, E. Devaux, and T. W. Ebbesen, Nano Lett. 9, 1278 (2009).

${ }^{22}$ R. F. Oulton, V. J. Sorger, T. Zentgraf, R. Ma, C. Gladden, L. Dai, G. Bartal, and X. Zhang, Nature (London) 461, 629 (2009).

${ }^{23}$ D. Martin-Cano, L. Martin-Moreno, F. J. Garcia-Vidal, and E. Moreno, Nano Lett. 10, 3129 (2010).

${ }^{24}$ A. Gonzalez-Tudela, D. Martin-Cano, E. Moreno, L. MartinMoreno, C. Tejedor, and F. J. Garcia-Vidal, Phys. Rev. Lett. 106, 020501 (2011). 
${ }^{25}$ K. J. Russell, T.-L. Liu, S. Cui, and E. L. Hu, Nat. Photonics 6, 459 (2012).

${ }^{26}$ Y. Gong and J. Vučković, Appl. Phys. Lett. 90, 033113 (2007).

${ }^{27}$ L. Novotny and B. Hecht, Principles of Nano-Optics (Cambridge University Press, Cambridge, UK, 2006).

${ }^{28}$ U. Weiss, Quantum Dissipative Systems (World Scientific, Singapore, 2008).

${ }^{29}$ B. Huttner and S. M. Barnett, Phys. Rev. A 46, 4306 (1992).

${ }^{30}$ T. G. Philbin, New J. Phys. 12, 123008 (2010).

${ }^{31}$ L. Knöll, S. Scheel, and D. Welsch, in Coherence and Statistics of Photons and Atoms, edited by J. Perina (Wiley, New York, 2001).

${ }^{32}$ H. T. Dung, L. Knöll, and D. G. Welsch, Phys. Rev. A 57, 3931 (1998).

${ }^{33}$ H. T. Dung, S. Y. Buhmann, L. Knöll, D. G. Welsch, S. Scheel, and J. Kästel, Phys. Rev. A 68, 043816 (2003).

${ }^{34}$ T. Gruner and D. G. Welsch, Phys. Rev. A 53, 1818 (1996).

${ }^{35}$ W. P. Schleich, Quantum Optics in Phase Space, 1st ed. (WileyVCH, Berlin, 2001), Chap. 14.

${ }^{36}$ D. Dzsotjan, A. S. Sørensen, and M. Fleischhauer, Phys. Rev. B 82, 075427 (2010).

${ }^{37}$ Y. Chen, T. R. Nielsen, N. Gregersen, P. Lodahl, and J. Mørk, Phys. Rev. B 81, 125431 (2010).

${ }^{38}$ T. Søndergaard and B. Tromborg, Phys. Rev. A 64, 033812 (2001).

${ }^{39}$ S. G. Rodrigo, F. J. Garcia-Vidal, and L. Martin-Moreno, Phys. Rev. B 77, 075401 (2008).

${ }^{40} \mathrm{C}$. Tai, Dyadic Green Functions in Electromagnetic Theory (Oxford University Press, New York, 1996), Chap. 2.

${ }^{41}$ G. W. Hanson and A. B. Yakovlev, Operator Theory for Electromagnetics: An Introduction (Springer, New York, 2002).

${ }^{42}$ A. J. Leggett, Phys. Rev. B 30, 1208 (1984).

${ }^{43}$ A. Garg, J. N. Onuchic, and V. Ambegaokar, J. Chem. Phys. 83, 4491 (1985).

${ }^{44}$ M. C. Goorden, M. Thorwart, and M. Grifoni, Phys. Rev. Lett. 93, 267005 (2004).

${ }^{45}$ L. Xian-Ting, Chem. Phys. Lett. 449, 296 (2007).

${ }^{46} \mathrm{H}$. Breuer and F. Petruccione, The Theory of Open Quantum Systems (Oxford University Press, Oxford, UK, 2002).

${ }^{47}$ C. Cohen-Tannoudji, J. Dupont-Roc, and G. Grynberg, AtomPhoton Interactions: Basic Processes and Applications (Wiley, New York, 1992), p. 680.

${ }^{48}$ D. Martin-Cano, A. González-Tudela, L. Martin-Moreno, F. J. Garcia-Vidal, C. Tejedor, and E. Moreno, Phys. Rev. B 84, 235306 (2011).

${ }^{49}$ A. Gonzalez-Tudela, P. A. Huidobro, L. Martin-Moreno, C. Tejedor, and F. J. Garcia-Vidal, arXiv:1205.3938 [Phys. Rev. Lett. (to be published)].

${ }^{50}$ R. R. Chance, A. Prock, and R. Silbey, Adv. Chem. Phys. 37, 1 (1978).

${ }^{51}$ W. L. Barnes, J. Mod. Opt. 45, 661 (1998).

${ }^{52}$ A. Auffèves, J. M. Gérard, and J. P. Poizat, Phys. Rev. A 79, 053838 (2009).

${ }^{53}$ A. Gonzalez-Tudela, E. del Valle, E. Cancellieri, C. Tejedor, D. Sanvitto, and F. P. Laussy, Opt. Express 18, 7002 (2010).

${ }^{54}$ A. Auffèves, D. Gerace, J. M. Gérard, M. F. Santos, L. C. Andreani, and J. P. Poizat, Phys. Rev. B 81, 245419 (2010).

${ }^{55}$ G. Cui and M. G. Raymer, Phys. Rev. A 73, 053807 (2006).
${ }^{56}$ A. Majumdar, A. Faraon, E. D. Kim, D. Englund, H. Kim, P. Petroff, and J. Vučković, Phys. Rev. B 82, 045306 (2010).

${ }^{57}$ E. Moreno, S. G. Rodrigo, S. I. Bozhevolnyi, L. Martin-Moreno, and F. J. Garcia-Vidal, Phys. Rev. Lett. 100, 023901 (2008).

${ }^{58}$ W. Wang, Q. Yang, F. Fan, H. Xu, and Z. L. Wang, Nano Lett. 11, 1603 (2011).

${ }^{59}$ D. J. Dikken, M. Spasenovi, E. Verhagen, D. van Oosten, and L. (Kobus) Kuipers, Opt. Express 18, 16112 (2010).

${ }^{60}$ R. F. Oulton, D. F. P. Pile, Y. Liu, and X. Zhang, Phys. Rev. B 76, 035408 (2007).

${ }^{61}$ K. J. Vahala, Nature (London) 424, 839 (2003).

${ }^{62}$ G. Rempe, R. J. Thompson, H. J. Kimble, and R. Lalezari, Opt. Lett. 17, 363 (1992).

${ }^{63}$ A. Laucht, N. Hauke, J. M. Villas-Bôas, F. Hofbauer, G. Böhm, M. Kaniber, and J. J. Finley, Phys. Rev. Lett. 103, 087405 (2009).

${ }^{64}$ I. Aharonovich, S. Castelletto, D. A. Simpson, C. H. Su, A. D. Greentree, and S. Prawer, Rep. Prog. Phys. 74, 076501 (2011).

${ }^{65}$ U. Banin, M. Bruchez, A. P. Alivisatos, T. Ha, S. Weiss, and D. S. Chemla, J. Chem. Phys. 110, 1195 (1999).

${ }^{66}$ A. V. Akimov, A. Mukherjee, C. L. Yu, D. E. Chang, A. S. Zibrov, P. R. Hemmer, H. Park, and M. D. Lukin, Nature (London) 450, 402 (2007).

${ }^{67}$ M. L. Andersen, S. Stobbe, A. S. Sorensen, and P. Lodahl, Nat. Phys. 7, 215 (2011).

${ }^{68}$ L. C. Andreani, G. Panzarini, and J. M. Gérard, Phys. Rev. B 60, 13276 (1999).

${ }^{69}$ F. P. Laussy, E. del Valle, and C. Tejedor, Phys. Rev. Lett. 101, 083601 (2008).

${ }^{70}$ E. del Valle, F. P. Laussy, and C. Tejedor, Phys. Rev. B 79, 235326 (2009).

${ }^{71}$ H. Ditlbacher, A. Hohenau, D. Wagner, U. Kreibig, M. Rogers, F. Hofer, F. Aussenegg, and J. Krenn, Phys. Rev. Lett. 95, 257403 (2005).

${ }^{72}$ W. L. Barnes, A. Dereux, and T. W. Ebbesen, Nature (London) 424, 824 (2003).

${ }^{73}$ Ernst Jan R. Vesseur, F. J. de Abajo, and A. Polman, Phys. Rev. B 82, 165419 (2010).

${ }^{74}$ M. P. Hiscocks, C. Su, B. C. Gibson, A. D. Greentree, L. C. L. Hollenberg, and F. Ladouceur, Opt. Express 17, 7295 (2009).

${ }^{75}$ D. Zueco, G. Reuther, S. Kohler, and P. Hänggi, Phys. Rev. A 80, 033846 (2009).

${ }^{76}$ K. Jacobs and A. Landahl, Phys. Rev. Lett. 103, 067201 (2009).

${ }^{77}$ A. Hoffman, S. Srinivasan, S. Schmidt, L. Spietz, J. Aumentado, H. Türeci, and A. Houck, Phys. Rev. Lett. 107, 053602 (2011).

${ }^{78}$ D. Angelakis, M. Santos, and S. Bose, Phys. Rev. A 76, 031805 (2007).

${ }^{79}$ T. Hümmer, G. Reuther, P. Hänggi, and D. Zueco, Phys. Rev. A 85, 052320 (2012).

${ }^{80}$ M. Mariantoni, H. Wang, T. Yamamoto, M. Neeley, R. C. Bialczak, Y. Chen, M. Lenander, E. Lucero, A. D. O’Connell, D. Sank, M. Weides, J. Wenner, Y. Yin, J. Zhao, A. N. Korotkov, A. N. Cleland, and J. M. Martinis, Science 334, 61 (2011).

${ }^{81}$ M. Hofheinz, H. Wang, M. Ansmann, R. C. Bialczak, E. Lucero, M. Neeley, A. D. O'Connell, D. Sank, J. Wenner, J. M. Martinis, and A. N. Cleland, Nature (London) 459, 546 (2009). 
${ }^{82}$ J. Majer, J. M. Chow, J. M. Gambetta, J. Koch, B. R. Johnson, J. A. Schreier, L. Frunzio, D. I. Schuster, A. A. Houck, A. Wallraff, A. Blais, M. H. Devoret, S. M. Girvin, and R. J. Schoelkopf, Nature (London) 449, 443 (2007).

${ }^{83}$ S. Diehl, A. Micheli, A. Kantian, B. Kraus, H. P. Büchler, and P. Zoller, Nat. Phys. 4, 878 (2008).
${ }^{84}$ F. Verstraete, M. M. Wolf, and J. Ignacio Cirac, Nat. Phys. 5, 633 (2009).

${ }^{85}$ W. Vogel and D.-G. Welsch, Quantum Optics (Wiley, New York, 2006), p. 520.

${ }^{86}$ F. Le Kien and K. Hakuta, Phys. Rev. A 80, 053826 (2009).

${ }^{87}$ D. Smith and F. Fickett, J. Res. Natl. Inst. Stand. Technol. 100, 119 (1995). 